\title{
Effet de la période et densité de semis sur la croissance et le rendement du cotonnier au Bénin
}

\author{
Isidore Amonmidé1*, Germain D. Fayalo1, Gustave D. Dagbenonbakin² \\ ${ }^{1}$ Centre de Recherches Agricoles Coton et Fibres, Institut National des Recherches Agricoles du Bénin (INRAB) \\ 2Institut National des Recherches Agricoles du Bénin (INRAB) \\ *Auteur correspondant : amonmide2018@yahoo.com; Tél. : (+229)97794507 / (+229)9584554, 01 BP 175 Cotonou \\ République du Bénin.
}

Original submitted in on $11^{\text {th }}$ May 2020. Published online at www.m.elewa.org/journals/ on $31^{\text {st }}$ August 2020

https://doi.org/10.35759/JABs.152.7

\section{RESUME}

Objectif: L'objectif de l'étude était d'identifier les meilleures périodes et densités de semis dans les différentes zones agro-écologiques cotonnières du Bénin dans un contexte de changement climatique.

Méthodologie et résultats : Les expérimentations ont été conduites pendant deux ans (2017 et 2018) en station au Bénin dans un dispositif expérimental en split-plot à deux facteurs, la période (facteur principal) et la densité de semis (facteur secondaire) respectivement à quatre et cinq variantes avec quatre répétitions. Les données collectées ont été soumises à une analyse de variance sous le logiciel R.3.6.1 au seuil de 5\% de probabilité d'erreur. Les résultats ont montré au cours des deux années d'expérimentation que les semis tardifs ont enregistré les plus faibles rendements en coton graine dans la zone centre-nord tandis que toutes les dates de semis ont donné des rendements équivalents dans la zone nord. La densité de semis à 62500 plants/ha a donné le meilleur rendement.

Conclusion et applications des résultats : Le semis du cotonnier peut s'étendre sur quatre décades (20 mai au 30 juin) dans la zone nord contrairement au centre-nord où la période optimale de semis s'étend seulement sur les deux dernières décades de juin (10-30 juin). La densité à 62500 plants/ha $(0,20 \mathrm{~m} \times 0,80 \mathrm{~m}$ à 1 plant/poquet) pourrait être recommandée pour l'amélioration des rendements en culture cotonnière au Bénin. L'adoption de cette densité de semis offre aux producteurs l'opportunité de mécaniser les opérations de semis et de fertilisation, compte tenu de la faible distance inter-poquets par rapport aux densités en vulgarisation.

Mots clés : Période de semis, densité de semis, rendement coton graine, zones cotonnières, Bénin.

Effect of sowing time and plant density on growth, development and yield in Benin

\section{ABSTRACT}

Objective: This study aimed at identifying the best sowing date and plant densities in the different cotton agro-ecological zones of Benin in a context of climate change.

Methodology and results: On-station trials were conducted during two years (2017 and 2018) in Benin in a split-plot experimental design with two factors, the sowing date (main factor) and the plant density (secondary factor) with four and five levels, respectively with four replications. Collected data were submitted to an analysis of variance under R.3.6.1 software at 5\% probability threshold error. Results showed other the two 
years of trial that late sowings recorded the lowest cotton seed yields in the northern central zone while all sowing date gave similar yields in the northern zone. Planting density of 62500 plants/ha gave the best yields. Conclusion and applications of the results: Cotton sowing could be extended over four decades (20 May to 30 June) in the northern zone contrarily to the northern central zone where the optimal sowing time covers only the last two decades of June (10-30 June). Plant density of 62500 plants/ha $(0.20 \mathrm{~m} \times 0.80 \mathrm{~m}$ at 1 plant/pot) could be recommended to improve cotton seed yields in Benin. The adoption of this new plant density offers producers the opportunity to mechanize sowing and fertilizer application, given low inter-plant space compared to the recommendation in Benin.

Key words: Sowing date, plant density, cotton seed yield, cotton agro-ecological zones, Benin.

\section{INTRODUCTION}

Le cotonnier (Gossypium hirsutum L.) est l'une des plantes à fibre les plus cultivées dans le monde. Au Bénin, comme dans la plupart des pays producteurs de coton, il constitue la première culture de rente et une importante source de devises (Arshad et al., 2007 ; PAM, 2014). II contribue à $45 \%$ des rentrées fiscales, $80 \%$ des recettes d'exportation, $13 \%$ de la formation du produit intérieur brut (PIB) national en termes de valeur ajoutée et $60 \%$ du tissu industriel (PNUD, 2011). La culture du coton constitue également la principale activité économique pour plus de 300000 ménages et procure un revenu monétaire à près de 2,5 millions de personnes ; soit $25 \%$ de la population (Banque Mondiale, 2016). II est cultivé dans la plupart des zones agroécologiques du Bénin (Hougni et al., 2016). Depuis la campagne 2016, les superficies emblavées sont en constante augmentation. Evaluées à 418 945,77 hectares en 2016, elles ont atteint un record de 656 463 hectares en 2018 (AIC, 2017, 2018). Aussi, les productions sont passées de 451 120,56 tonnes à 678000 tonnes au cours de la même période (AIC, 2017, 2018). Malgré l'amélioration de la production ces dernières années, les rendements sont toujours faibles $(1070 \mathrm{~kg} / \mathrm{ha}$ de coton non égrené en 2018) (AIC, 2020) comparativement aux grands pays producteurs de coton comme la Chine $(5280,2$ $\mathrm{kg} / \mathrm{ha}$ ), les Etats Unis d'Amérique (2682,1 kg/ha), le Pakistan (2034,8 kg/ha de coton non égrené) en 2018 (FAOSTAT, 2020). Le rendement moyen national est passé de $716 \mathrm{~kg} / \mathrm{ha}$ (en 2012) à 1128 $\mathrm{kg} / \mathrm{ha}$ de coton graine en 2017 (INSAE, 2019) alors qu'il peut atteindre 2 à 4 tonnes par hectare comme c'est le cas en Chine, en Inde et au Brésil. A part la baisse continue de la fertilité des sols dans les zones cotonnières et la pression parasitaire rapportées par plusieurs études au Bénin (Igue et al., 2013, Djihinto et al., 2016 ; Amonmidé et al., 2019), d'autres facteurs peuvent justifier les faibles rendements observés. II s'agit notamment de la date et la densité de semis qui conditionnent le rendement de la culture (Arshad et al., 2007; Dong et al., 2012; Yang et al., 2014). Les perturbations climatiques de ces dernières années qui se manifestent par des poches de sécheresse en début de campagne cotonnière ne facilitent plus les semis à bonne date du cotonnier entrainant un étalement des semis sur une période plus longue. Lorsque les semis précoces et les semis à bonne date améliorent le nombre et le poids des capsules et le rendement en coton graine (Arshad et al., 2007), les semis tardifs par contre affectent négativement ces paramètres et le rendement (Ali et al., 2004; Bilal et al., 2015). La prévalence de conditions environnementales défavorables à la croissance, la floraison, la formation des capsules et leur maintien constituent les causes de différence de performance observée entre un semis précoce et un semis tardif (Kakar et al., 2012). Par ailleurs, la densité de semis constitue un facteur primordial dans l'amélioration de la productivité du coton (Soomro et al., 2000 ; Khan et al., 2005; Yang et al., 2014). Une très faible ou très forte densité de semis peut compromettre le rendement de la culture (Ciampitti \& Vyn, 2011). Ainsi, Soomro et al. (2000) ont rapporté qu'un écartement de 23 et $30 \mathrm{~cm}$ entre plants ont enregistré un rendement en coton graine supérieur à un écartement de 15 et $38 \mathrm{~cm}$ et ont recommandé un écartement de $20-30 \mathrm{~cm}$ entre plants au Pakistan. Khan et al. (2005) ont rapporté une densité de 100000 plants par hectare comme optimale avec un écartement entre lignes de 0,76 
m. Cependant, Jagannathan \& Venkitaswamy (1996) ont rapporté une diminution de rendement de coton graine avec l'augmentation de la densité de semis bien que d'autres auteurs aient rapporté l'inexistence de relation entre la densité de semis et le rendement de coton graine (Muhammad et al., 2002; Akhtar et al., 2003 ; Shah et al., 2005). Depuis 2017, des essais de périodes et de densités de semis ont été initiés par le Centre de Recherches Agricoles Coton et Fibres (CRA-CF) de l'Institut National des Recherches Agricoles du Bénin (INRAB) afin de proposer des approches de solutions aux producteurs dans le contexte actuel

\section{MATERIEL ET METHODES}

Milieu d'étude : Les expérimentations ont été conduites sur deux (2) centres permanents d'expérimentations (CPE) du Centre de Recherches Agricoles Coton et Fibres (CRA-CF) de l'Institut National des Recherches Agricoles du Bénin (INRAB). II s'agit des CPE de Angaradébou ( $3^{\circ} 1^{\prime} 00^{\prime \prime} \mathrm{E}, 11^{\circ} 19^{\prime} 50^{\prime \prime} \mathrm{N}$ ) et de Okpara $\left(9^{\circ} 20^{\prime} 49^{\prime \prime} \mathrm{E}, 2^{\circ} 41^{\prime} 3^{\prime \prime} \mathrm{N}\right)$ représentant respectivement les zones nord et centre-nord. Ces deux zones produisent plus de $70 \%$ du coton au plan national (AIC, 2017, 2018). Matériel végétal : L'évaluation des différentes périodes et densités de semis a été effectuée sur deux variétés de cotonnier. Chacune des variétés est spécifique à une zone de production. Les variétés ANG 956 et OKP 768 ont été utilisées respectivement à Angaradébou et à Okpara.

Fertilisation et protection phytosanitaire: La fertilisation était de type minéral avec l'engrais coton $14 \mathrm{~N}-18 \mathrm{P}-18 \mathrm{~K}+6 \mathrm{~S}+1 \mathrm{~B}$ à la dose de $200 \mathrm{~kg} / \mathrm{ha}$. de changement climatique. Ainsi, l'objectif général de la présente étude était de réévaluer les périodes et densités de semis en recommandation en lien avec de nouvelles propositions afin d'identifier les meilleures périodes et densités de semis dans les différentes zones agro-écologiques cotonnières du Bénin dans un contexte de changement climatique. Deux objectifs spécifiques ont été fixés : i) évaluer l'efficacité des semis précoces, tardifs et densités de semis sur le rendement en coton graine, ii) évaluer l'efficacité des semis précoces, tardifs et densités de semis sur la morphologie du cotonnier.

L'application a été réalisée à 15 jours après semis (JAS). La fumure d'entretien était à base d'urée (46\% d'azote) à la dose de $50 \mathrm{~kg} / \mathrm{ha}$ à $45 \mathrm{JAS}$.

Trois catégories d'insecticides ont été utilisées pour la protection phytosanitaire des plants de cotonnier. La première catégorie était à base d'émamectine, la seconde à base de binaire acaricide, et la troisième à base de binaire aphicide.

Dispositif expérimental: Le dispositif expérimental était en split-plot à deux facteurs, la période (facteur principal) et la densité de semis (facteur secondaire) à 4 et 5 niveaux (Tableau 1), respectivement. Le nombre de répétition par traitement était 4 . Les parcelles élémentaires étaient constituées de 4 lignes de $9 \mathrm{~m}$. L'écartement entre lignes était de $0,80 \mathrm{~m}$. La superficie d'une parcelle élémentaire était de $28,8 \mathrm{~m}^{2}(9 \mathrm{~m} \times 0,8 \mathrm{~m}$ $x 4$ ).

Tableau 1 : Périodes et densités de semis évaluées

\begin{tabular}{|c|c|}
\hline \multicolumn{2}{|c|}{ Périodes de semis (2017 et 2018) } \\
\hline Zone nord & Zone centre-nord \\
\hline $\mathrm{P} 1=20-30$ mai & $P 1=1-10$ juin \\
\hline P2 = 1-10 juin & $P 2=11-20$ juin \\
\hline$P 3=11-20$ juin & $\mathrm{P} 3=21-30$ juin \\
\hline$P 4=21-30$ juin & P4 $=1-10$ juillet \\
\hline $\begin{array}{l}D 1=416 \\
D 2=625 \\
D 3=625 \\
D 4=500 \\
D 5=416\end{array}$ & $\begin{array}{l}\text { plant/poquet) } \\
\text { plants/poquet) } \\
\text { plant/poquet) } \\
\text { plants/poquet) } \\
\text { plants/poquet) }\end{array}$ \\
\hline
\end{tabular}


Données collectées : A 30 jours après levée (JAL), dix (10) plants dont cinq (5) sur chacune des deux lignes centrales ont été marqués à l'aide de ficelle. La hauteur des cotonniers a été prise par plant marqué à l'aide d'une règle graduée $(\mathrm{cm})$ à partir du premier nœud cotylédonaire. A 60 et $90 \mathrm{JAL}$, les mesures de hauteur ont été répétées sur les mêmes plants préalablement marqués. La hauteur a une grande influence sur la mise en place des organes de reproduction. Le shedding des organes fructifères (boutons floraux et capsules) a été effectué hebdomadairement dans la première interligne à partir de 60 jours après levée (JAL) jusqu'à 120 JAL. Après la récolte, le plant mapping a été réalisé sur dix (10) plants dont cinq (5) sur chacune des deux lignes centrales. Le plant mapping concerne le niveau d'insertion de la première branche fructifère (NiPBF), le

\section{RESULTATS}

Evolution de la pluviométrie au cours des essais : Les figures 1 et 2 présentent l'évolution de la pluviosité au cours des essais en 2017 et 2018 sur les deux sites ayant abrité les expérimentations. Globalement, la pluviométrie a été satisfaisante et bien répartie tout au long du cycle cultural du cotonnier. Une très faible variation a été observée entre le cumul pluviométrique du semis à la récolte entre 2017 (970,2 mm; Figure 2a) et 2018 (1131,5 mm; Figure 2b) à Angaradébou contrairement à Okpara où la différence inter annuelle a été plus importante. En effet, le cumul pluviométrique enregistré du semis à la récolte en 2017 (1056,5 mm ; nombre de branches végétatives (NBV) et le nombre de branches fructifères (NBF). Ces composantes du rendement sont déterminantes dans l'amélioration du rendement. A l'ouverture complète des capsules, les deux lignes centrales de chaque parcelle élémentaire ont été récoltées. Le coton graine a été pesé, et le rendement a été évalué.

Analyses statistiques : Les données agronomiques et morphologiques collectées ont été soumises à une analyse de la variance à deux critères de classification sous le logiciel R.3.6.1. L'uniformité de la distribution des données a été vérifiée avant les analyses. Le test de comparaison par paire (pairwise.t.test) a été utilisé pour la séparation des moyennes significativement différentes à une probabilité de 5\% d'erreur.

Figure 1a) a été plus important que celui enregistré en 2018 (773 mm ; Figure 1b). Au total 44 et 54 jours pluvieux $(\geq 1 \mathrm{~mm})$ ont été enregistrés à Okpara respectivement en 2017 et en 2018 avec six (6) et deux (2) hauteurs d'eau supérieures à $50 \mathrm{~mm}$ (Figure 1). Cependant, le nombre de jours de pluie de la période a été plus élevé à Angaradébou, 50 (2017) et 62 (2018) avec seulement trois (3) et quatre (4) importantes hauteurs d'eau ( $\geq 50 \mathrm{~mm}$ ), respectivement en 2017 et 2018 (Figure 2).

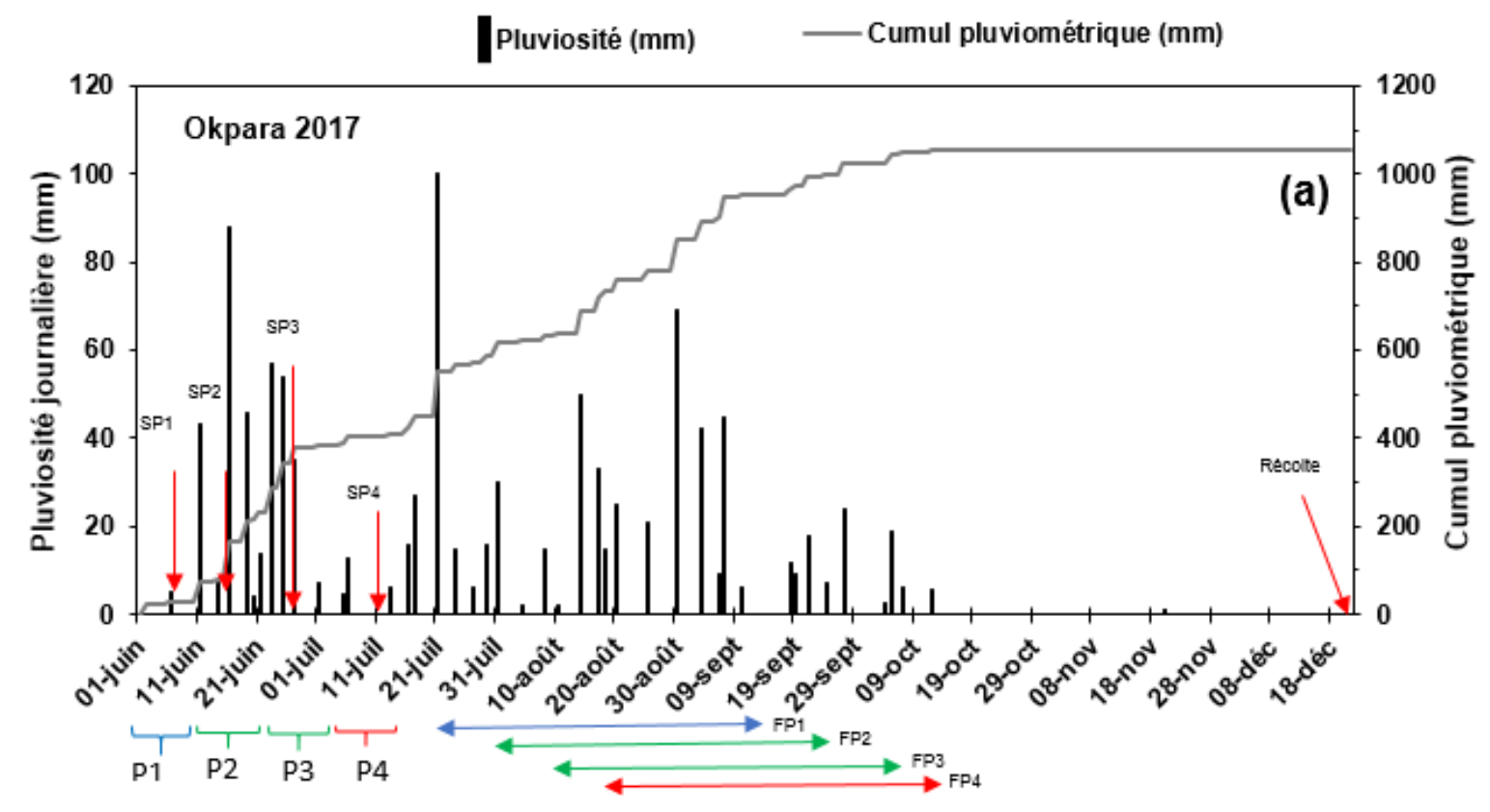




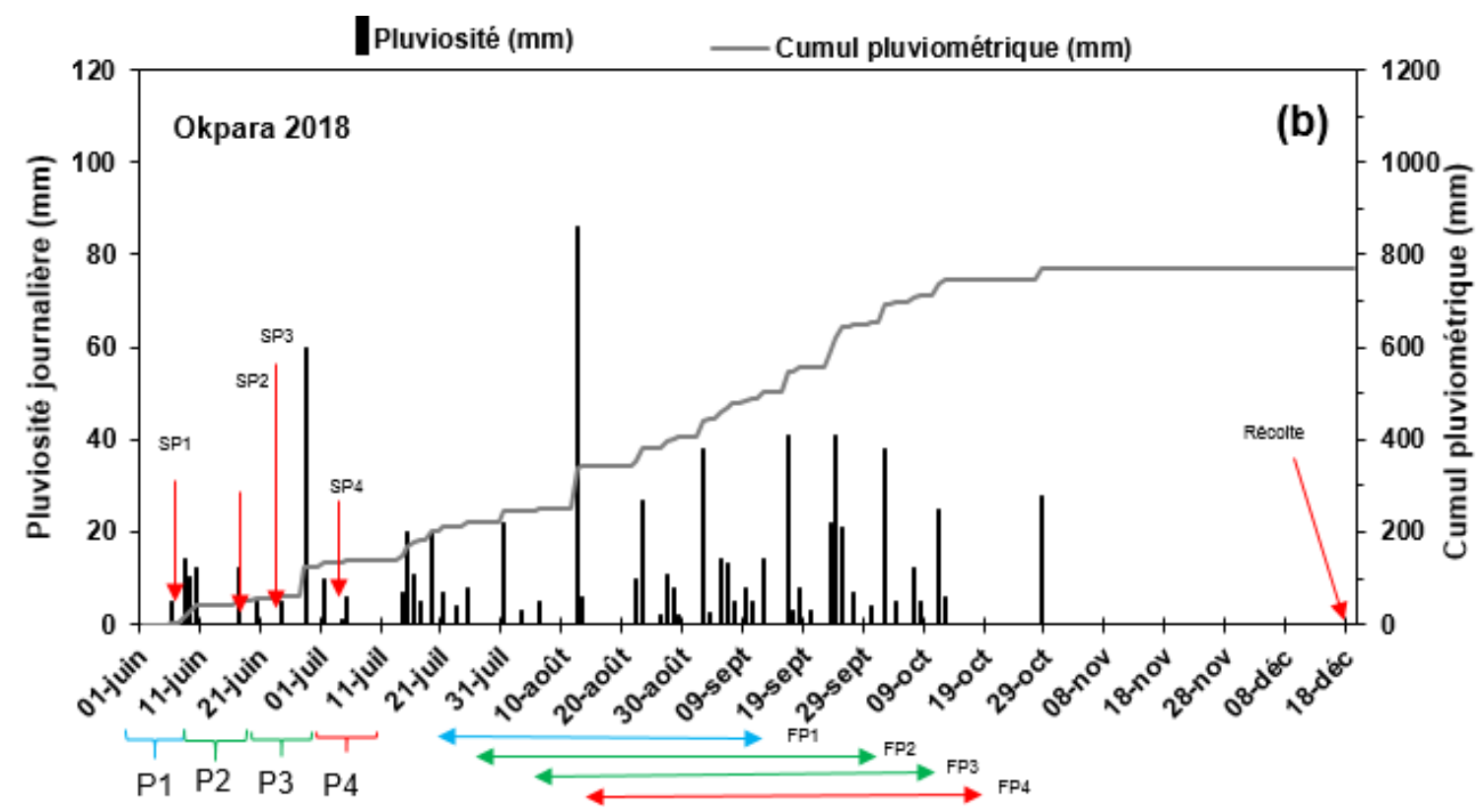

Figure 1 : Evolution de la pluviométrie au cours des expérimentations sur le site de Okpara en 2017 (a) et 2018 (b). Légende : Sp1, SP2, Sp3 et Sp4 désignent les dates de semis du cotonnier au cours des différentes périodes testées. Fp1, FP2, Fp3 et Fp4 désignent les périodes de floraison du cotonnier pour les différentes périodes de semis testées. $P 1, P 2, P 3$ et P4 représentent les périodes de semis entre 1-10 juin, 11-20 juin, 21-30 juin et 1-10 juillet (2017 et 2018), respectivement.

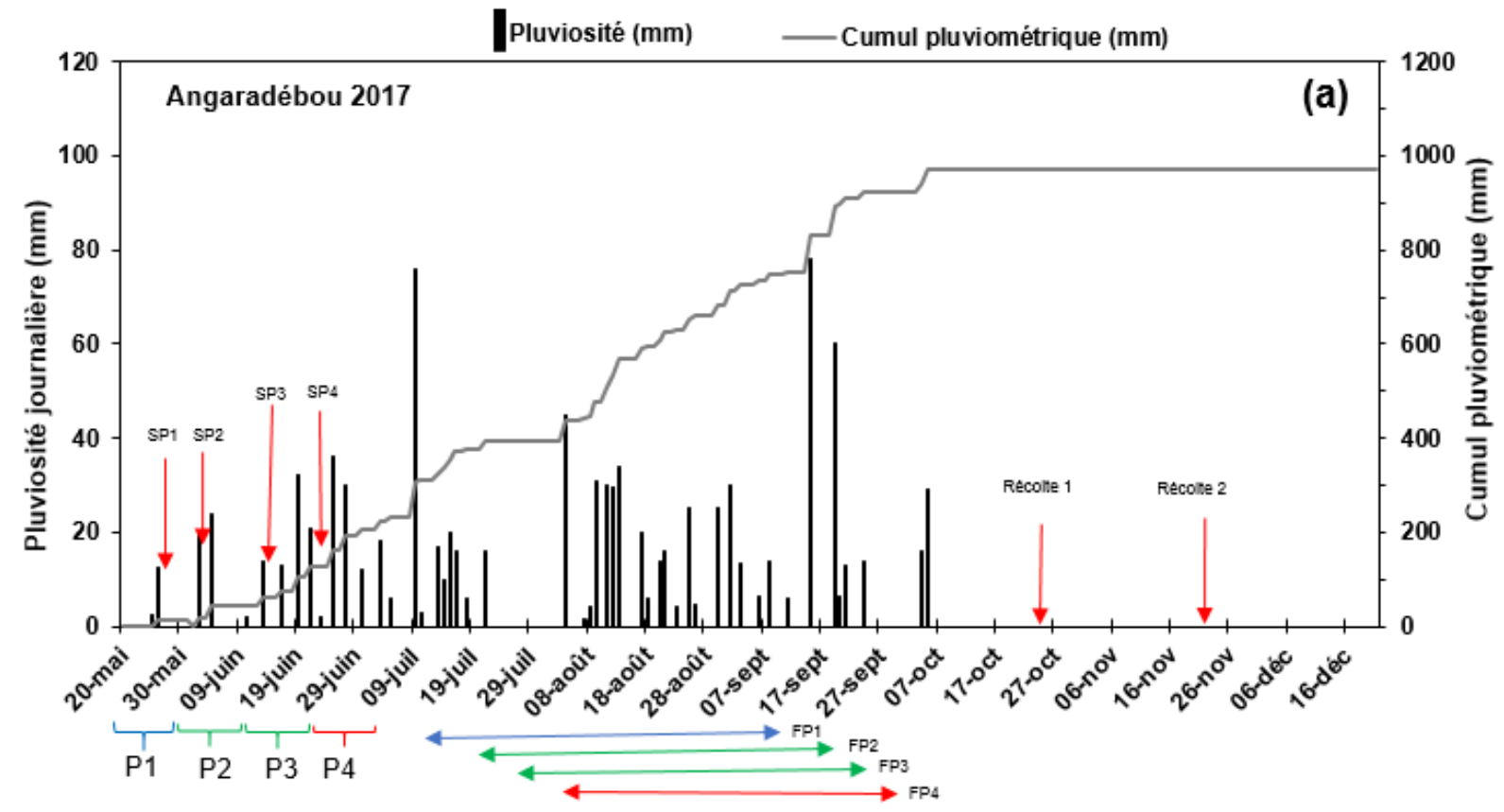




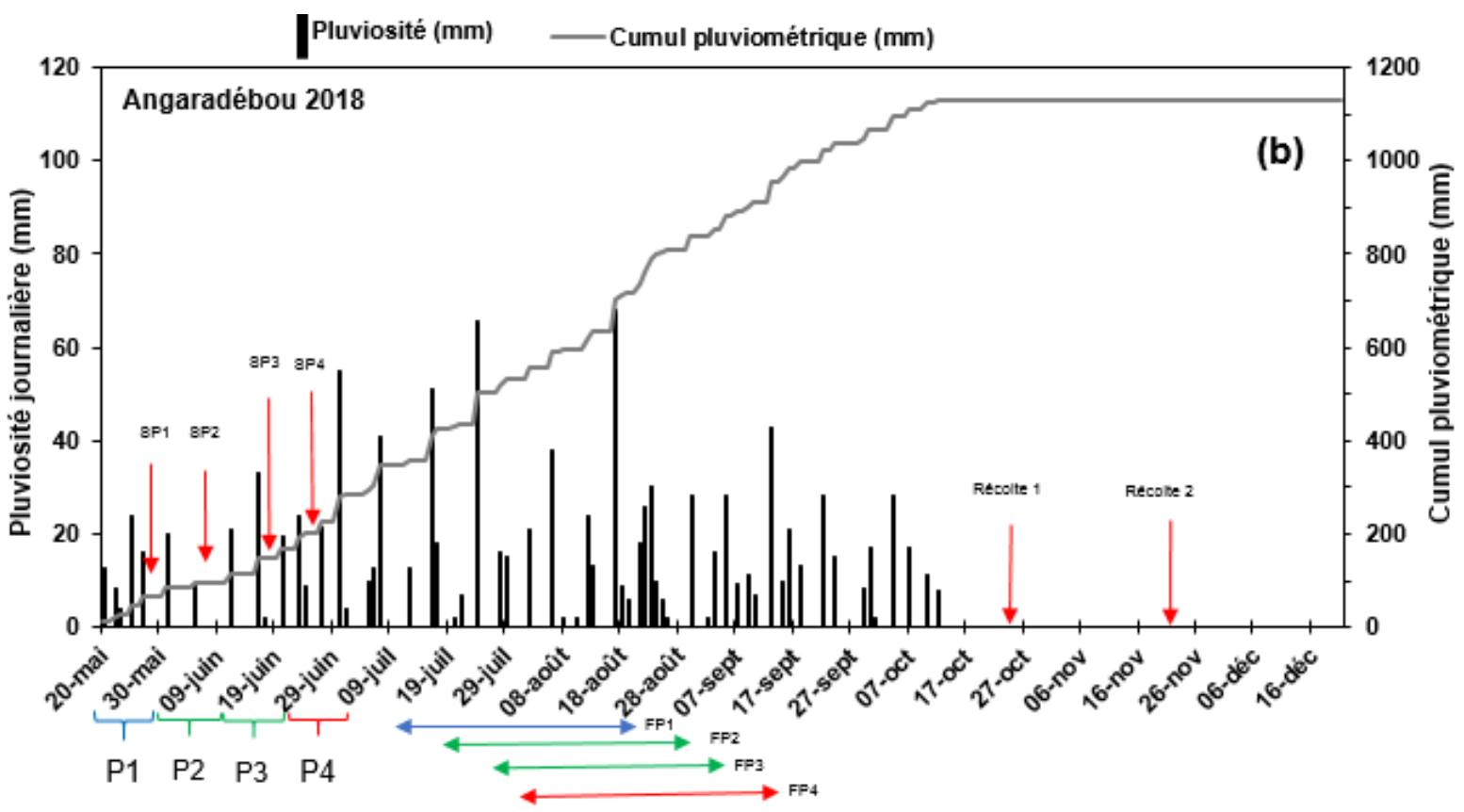

Figure 2 : Evolution de la pluviométrie au cours des expérimentations sur le site de Angaradébou en 2017 (a) et 2018 (b)

Légende : Sp1, SP2, Sp3 et Sp4 désignent les dates de semis du cotonnier au cours des différentes périodes testées.

$\mathrm{Fp} 1, \mathrm{FP} 2, \mathrm{Fp} 3$ et Fp4 désignent les périodes de floraison du cotonnier pour les différentes périodes de semis testées.

$P 1, P 2, P 3$ et P4 représentent les périodes de semis entre 20-30 mai, 1-10 juin, 11-20 juin et 21-30 juin (2017 et 2018), respectivement.

Effet de la période de semis sur le rendement en coton graine au Bénin : Pour l'ensemble des deux sites, les meilleurs rendements ont été obtenus en 2017. De plus, au cours de la même campagne, en dehors de la quatrième période, les rendements, ont été plus importants à Okpara. Les analyses statistiques n'ont montré un effet significatif de la période de semis sur le rendement en coton graine que sur le site de Okpara en $2017(P=0,000$; Tableau 2), en $2018(P=0,000$; Tableau 2) et pour l'ensemble des deux années d'essai $(P=0,000$; Tableau 3). Les semis au cours de la première et la deuxième décade du mois de juin ( $\mathrm{P} 1$ et P2) ont enregistré les rendements en coton graine les plus élevés comparativement aux autres périodes (Figures 3 \& 4). En 2017, P1 et P2 ont produit en moyenne $3118,75 \pm 435,6$ et $2931,25 \pm 455,32 \mathrm{~kg} / \mathrm{ha}$ de coton graine respectivement contre 2645,49 \pm 419,57 $\mathrm{kg} / \mathrm{ha}$ pour P3 et $1467,01 \pm 384,80 \mathrm{~kg} / \mathrm{ha}$ pour P4 (Figure 3). Une amélioration de rendement de 18 et $113 \%$ a été obtenue par P1 comparativement à P3 et P4, respectivement. En 2018, P2 a enregistré le meilleur rendement de coton graine $(2611,98 \pm 356,49 \mathrm{~kg} / \mathrm{ha})$ comparativement aux autres, soit une amélioration de rendement de $20 ; 18$ et $109 \%$ par rapport à $\mathrm{P} 1, \mathrm{P} 3$ et P4, respectivement (Figure 3). Pour l'ensemble des deux années d'essai, P1 et P2 ont donné des rendements équivalents mais significativement meilleurs à P3 et P4 (Figure 4). Cependant à Angaradébou, les rendements en coton graine observés ne différaient pas significativement entre les différentes périodes de semis testées en 2017, 2018 et pour l'ensemble des deux années d'essai $(P>0,05$; Tableaux $2 \& 3)$. Les rendements de coton graine obtenus par période étaient de : 2286,11 \pm 403,02 (P1), 1952,78 \pm 580,92 (P2) ; $2399,48 \pm 484,37$ (P3) et 2061,81 $\pm 248,36 \mathrm{~kg} / \mathrm{ha}$ (P4) en $2017 ; 2217,36 \pm 391,26 ; 1825,69 \pm 492,08$; $1738,19 \pm 445,75 ; 2054,55 \pm 472,67 \mathrm{~kg} / \mathrm{ha}$ en 2018 et $2251,74 \pm 393,60 ; 1889,24 \pm 535,27 ; 2068,84 \pm$ 568,$53 ; 2058,18 \pm 372,71 \mathrm{~kg} / \mathrm{ha}$ pour l'ensemble des deux années, respectivement (Figures $5 \&$ et 6 ). 


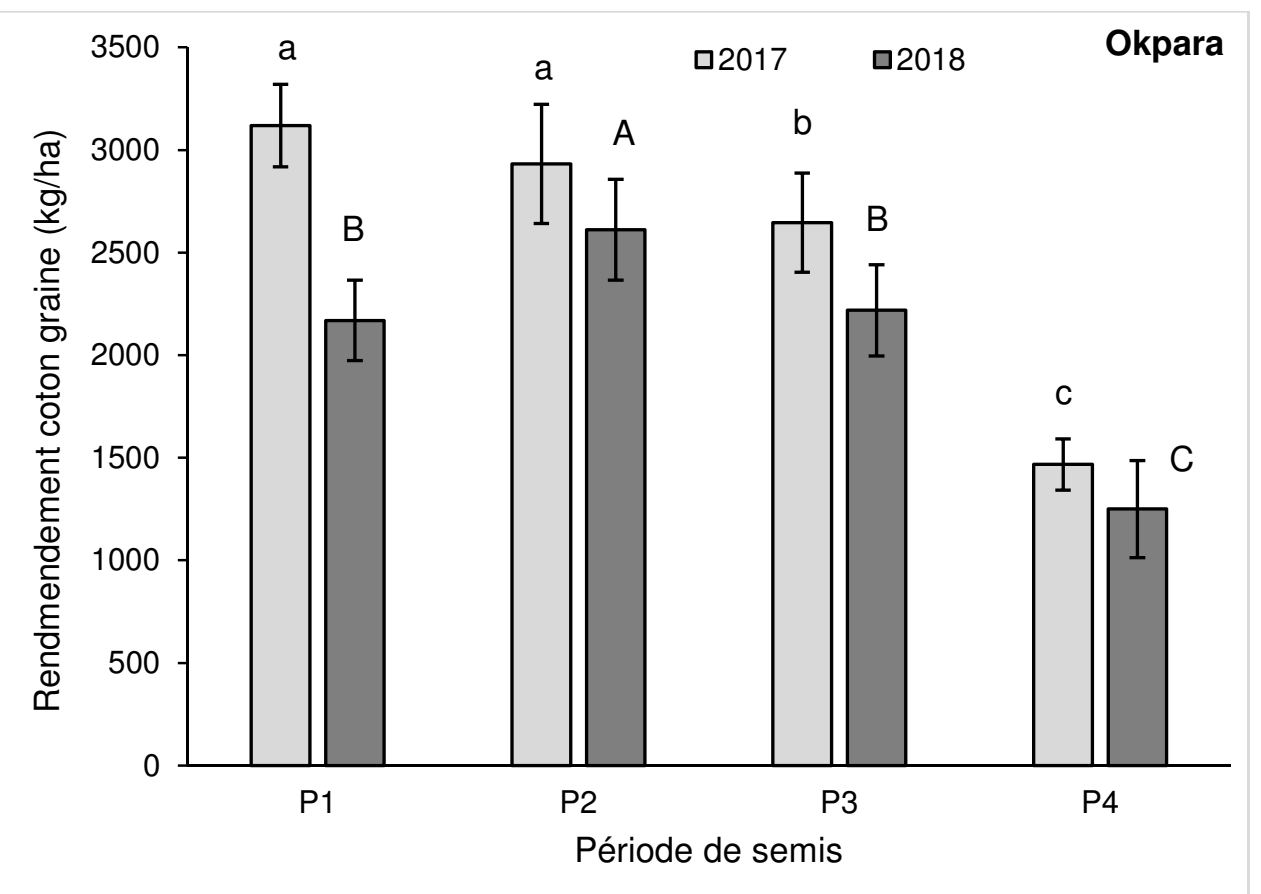

Figure 3: Rendement coton graine en fonction de la période de semis à Okpara au cours des deux années d'expérimentation.

Légende : P1, P2, P3 et P4 représentent les périodes de semis entre 1-10 juin, 11-20 juin, 21-30 juin et 1-10 juillet (2017 et 2018), respectivement. Les barres d'erreur représentent l'écart type. Les moyennes suivies des mêmes lettres et de même caractère ne sont pas significativement différentes au seuil de $5 \%$.

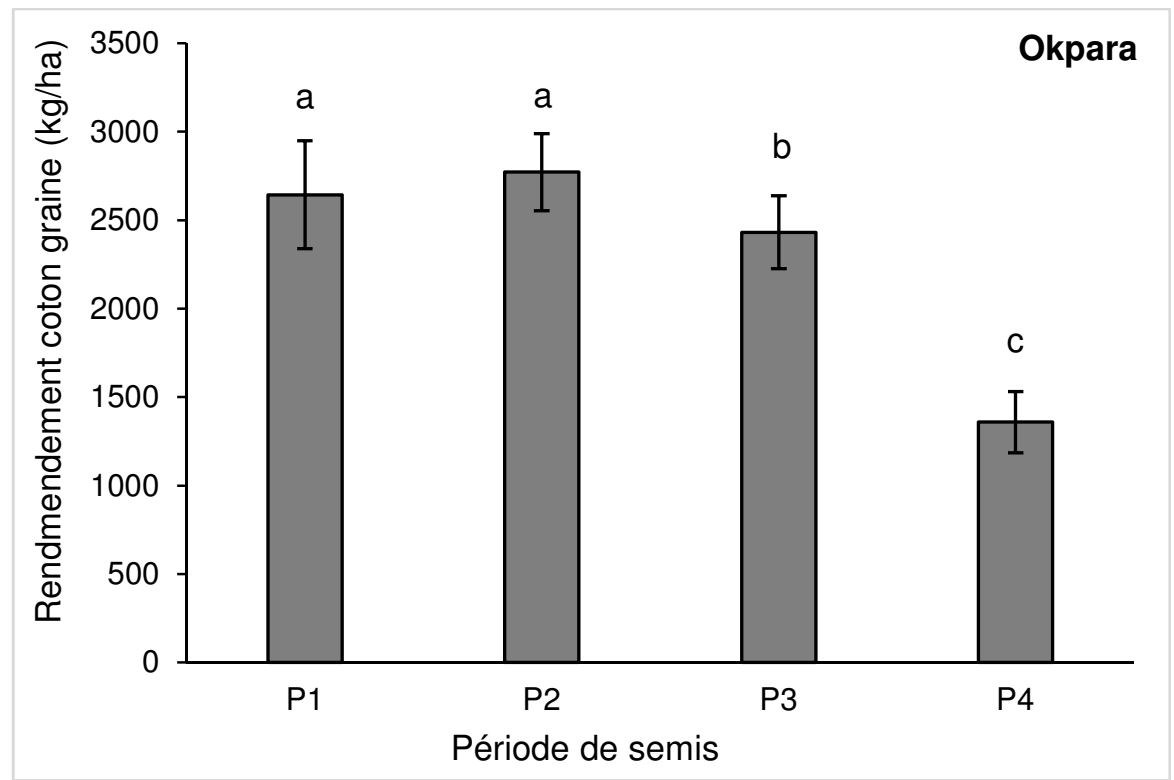

Figure 4 : Rendement coton graine en fonction de la période de semis à Okpara pour l'ensemble des deux années d'expérimentation.

Légende : P1, P2, P3 et P4 représentent les périodes de semis entre 1-10 juin, 11-20 juin, 21-30 juin et 1-10 juillet (2017 et 2018), respectivement. Les barres d'erreur représentent l'écart type. Les moyennes suivies des mêmes lettres ne sont pas significativement différentes au seuil de $5 \%$. 


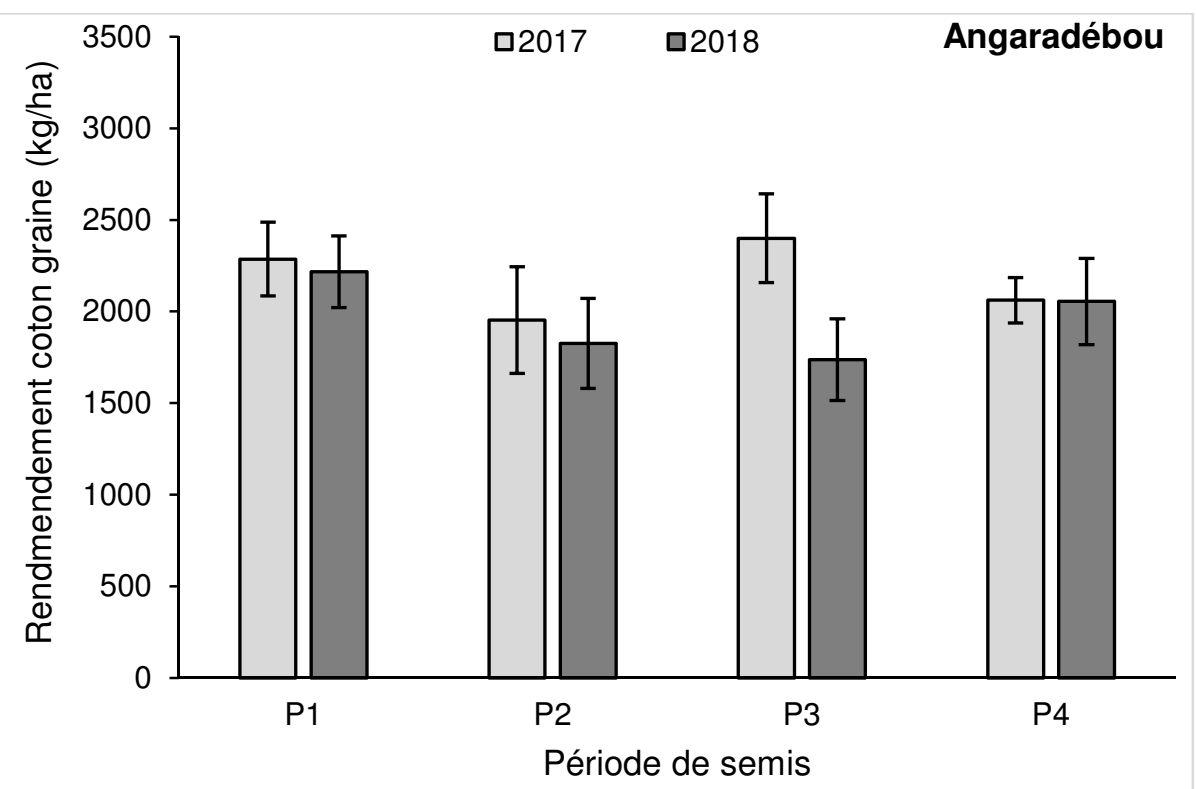

Figure 5 : Rendement coton graine en fonction de la période de semis à Angaradébou au cours des deux années d'expérimentation.

Légende : P1, P2, P3 et P4 représentent les périodes de semis entre 20-30 mai, 1-10 juin, 11-20 juin et 21-30 juin (2017 et 2018), respectivement. Les barres d'erreur représentent l'écart type.

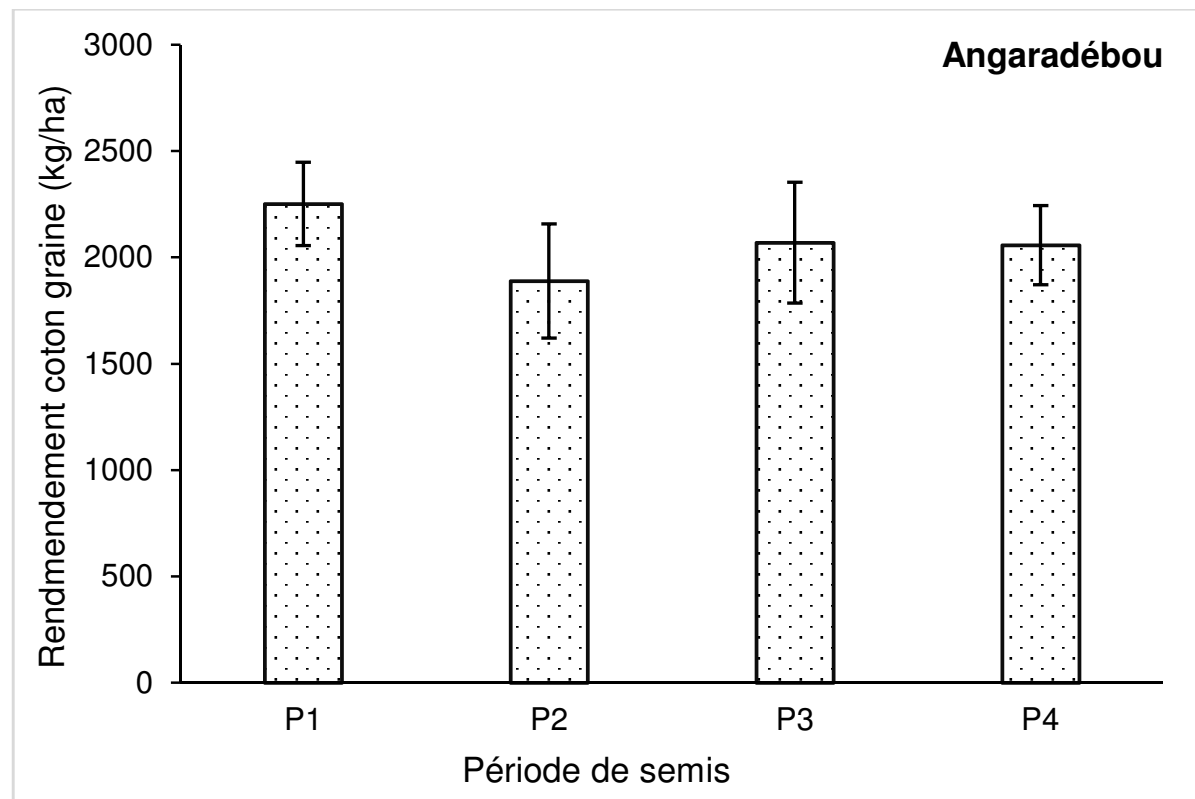

Figure 6 : Rendement coton graine en fonction de la période de semis à Angaradébou pour l'ensemble des deux années d'expérimentation.

Légende : P1, P2, P3 et P4 représentent les périodes de semis entre 20-30 mai, 1-10 juin, 11-20 juin et 21-30 juin, respectivement. Les barres d'erreur représentent l'écart type par rapport à la moyenne.

Effet de la densité de semis sur le rendement en coton graine au Bénin: A Angaradébou, les rendements ont été plus importants en 2017 pour les densités D1 (41667 plants/ha $(0,30 \mathrm{~m} \times 0,80 \mathrm{~m}$ à 1 plant/poquet)) et D2 (62500 plants/ha $(0,40 \mathrm{~m} \times 0,80 \mathrm{~m}$ à 2 plants/poquet)). Par contre en 2018, le meilleur rendement a été obtenu sous D3 (62500 plants/ha (0,20 $\mathrm{m} \times 0,80 \mathrm{~m}$ à 1 plant/poquet; Figure 7)). Si en 2018, l'analyse statistique a montré de différence significative $(P=0,005 ;$ Tableau 2) entre les densités de semis, 
aucune influence positive de la densité de semis n'a été observée sur rendement en 2017 ( $P>0,05$; Tableau 2 ; Figure 7) et pour l'ensemble des deux années d'essai (Figure 8). A Okpara, en 2017, les meilleurs rendements ont été obtenus sous les densités D3, D1 et D2. Ces densités ont été statistiquement équivalentes et différentes de D4 et D5 (Figure 9). Par contre, en 2018, les cinq densités ont donné des rendements équivalents (Figure 9). Pour l'ensemble des deux années d'expérimentation, le rendement moyen le plus important a été obtenu avec la densité D3 (62500 plants/ha $(0,20 \mathrm{~m} \times 0,80 \mathrm{~m}$ à 1 plant/poquet ; Figure 10)). L'analyse statistique des rendements moyens n'a pas montré de différence significative entre les différentes densités $(P>0,05$; Tableaux 2 et 3$)$. Par ailleurs, aucune interaction significative n'a été observée entre les périodes et densités de semis en 2017, 2018 et pour l'ensemble des deux années sur les différents sites $(P>$ $0,05$; Tableaux 2 et 3$)$.

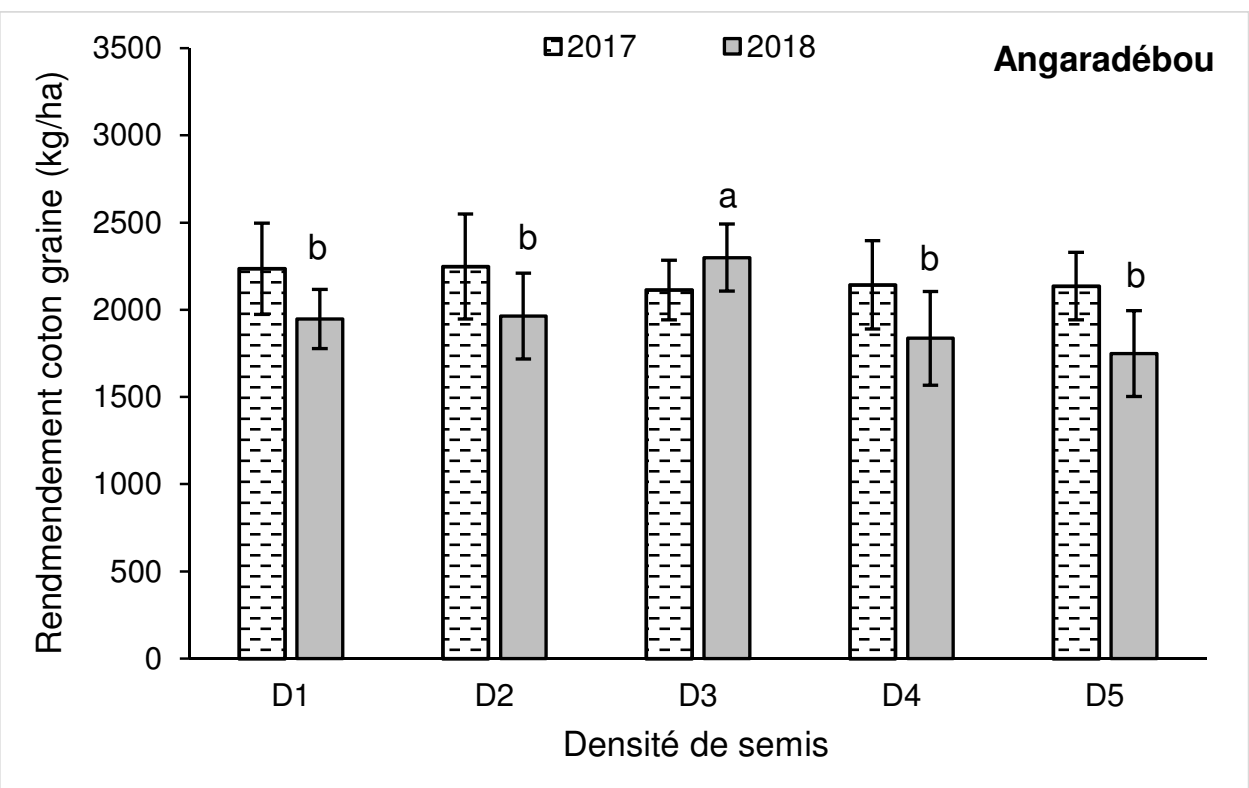

Figure 7 : Rendement coton graine en fonction de la densité de semis dans la zone nord (Angaradébou) au cours des deux années d'expérimentation.

Légende : D1, D2, D3, D4 et D5 représentent les densités de semis à 41667 plants/ha $(0,30 \mathrm{~m} \times 0,80 \mathrm{~m}$ à 1 plant/poquet, 62500 plants/ha $(0,40 \mathrm{~m} \times 0,80 \mathrm{~m}$ à 2 plants/poquet), 62500 plants/ha $(0,20 \mathrm{~m} \times 0,80 \mathrm{~m}$ à 1 plant/poquet), 50000 plants/ha $(0,50 \mathrm{~m} \times 0,80$ $\mathrm{m}$ à 2 plants/poquet) et 41667 plants/ha $(0,60 \mathrm{~m} \times 0,80 \mathrm{~m}$ à 2 plants/poquet), respectivement. Les barres d'erreur représentent l'écart type par rapport à la moyenne. Les moyennes suivies des mêmes lettres ne sont pas significativement différentes au seuil de $5 \%$. 


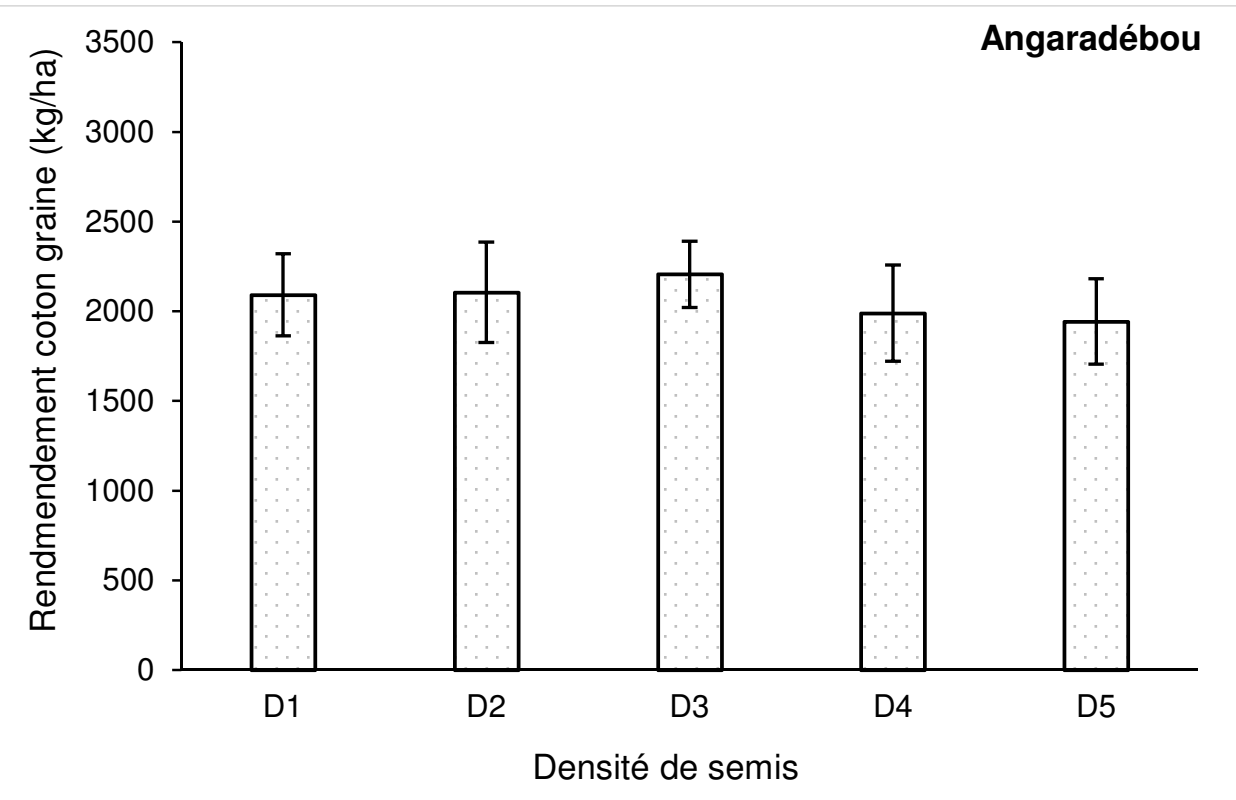

Figure 8 : Rendement coton graine en fonction de la densité de semis à Angaradébou pour l'ensemble des deux années d'expérimentation.

Légende : D1, D2, D3, D4 et D5 représentent les densités de semis à 41667 plants/ha $(0,30 \mathrm{~m} \times 0,80 \mathrm{~m}$ à 1 plant/poquet, 62500 plants/ha (0,40 $\mathrm{m} \times 0,80 \mathrm{~m}$ à 2 plants/poquet), 62500 plants/ha $(0,20 \mathrm{~m} \times 0,80 \mathrm{~m}$ à 1 plant/poquet), 50000 plants/ha $(0,50 \mathrm{~m} \times 0,80$ $\mathrm{m}$ à 2 plants/poquet) et 41667 plants/ha $(0,60 \mathrm{~m} \times 0,80 \mathrm{~m}$ à 2 plants/poquet), respectivement. Les barres d'erreur représentent l'écart type par rapport à la moyenne.

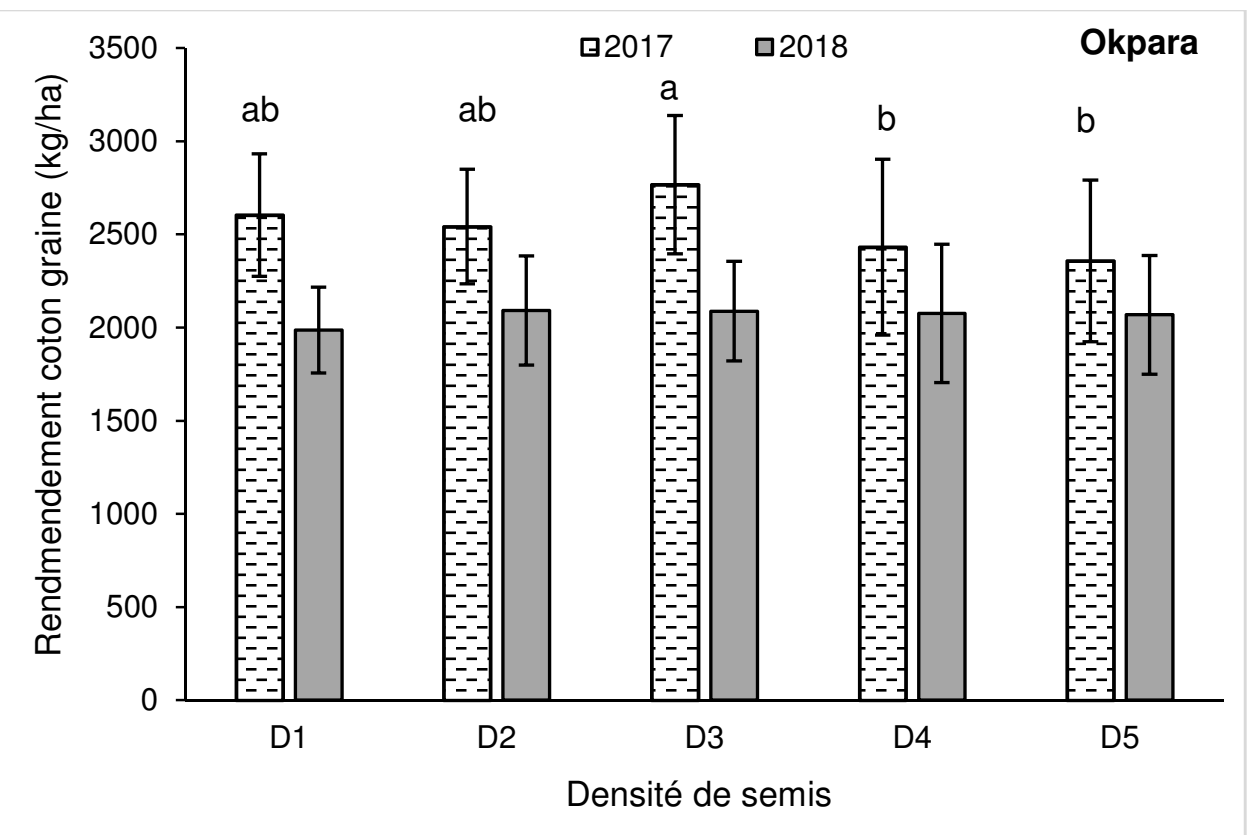

Figure 9: Rendement coton graine en fonction de la densité de semis à Okpara au cours des deux années d'expérimentation.

Légende : D1, D2, D3, D4 et D5 représentent les densités de semis à 41667 plants/ha $(0,30 \mathrm{~m} \times 0,80 \mathrm{~m}$ à 1 plant/poquet, 62500 plants/ha $(0,40 \mathrm{~m} \times 0,80 \mathrm{~m}$ à 2 plants/poquet), 62500 plants/ha $(0,20 \mathrm{~m} \times 0,80 \mathrm{~m}$ à 1 plant/poquet), 50000 plants/ha $(0,50 \mathrm{~m} \times 0,80$ $\mathrm{m}$ à 2 plants/poquet) et 41667 plants/ha $(0,60 \mathrm{~m} \times 0,80 \mathrm{~m}$ à 2 plants/poquet), respectivement. Les barres d'erreur représentent l'écart type par rapport à la moyenne. Les moyennes suivies des mêmes lettres ne sont pas significativement différentes au seuil de $5 \%$. 


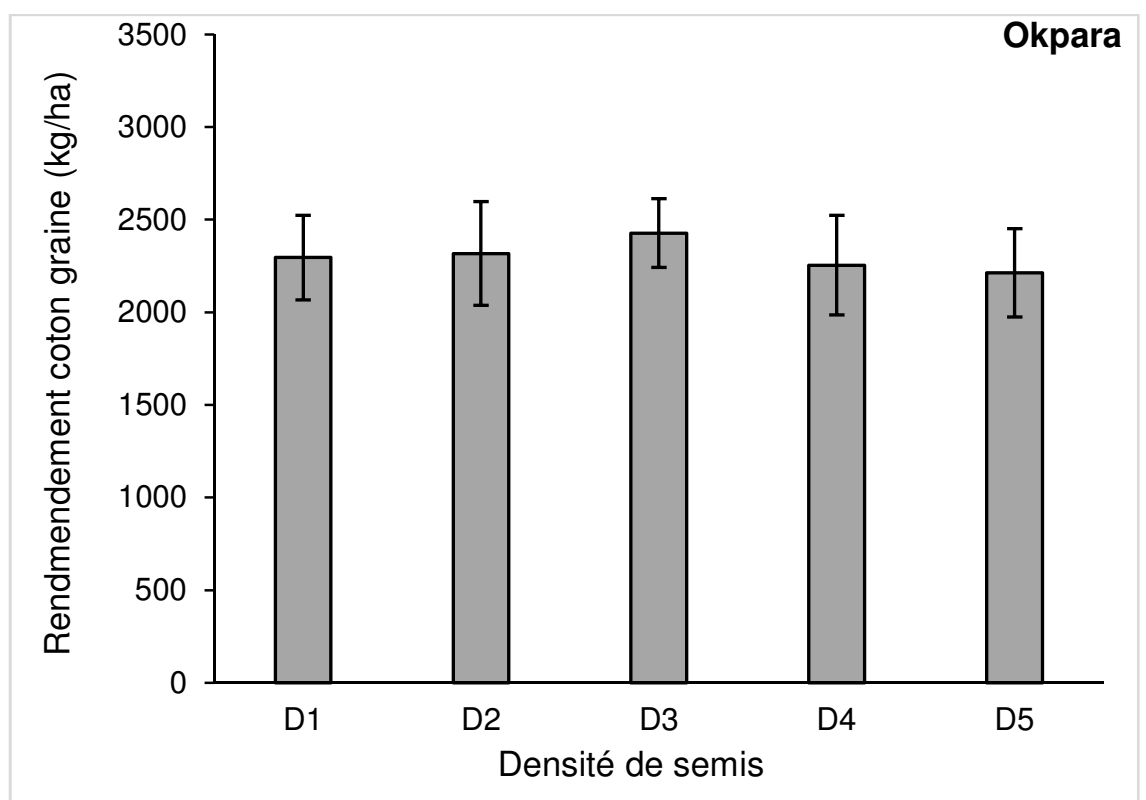

Figure 10 : Rendement coton graine en fonction de la densité de semis à Okpara pour l'ensemble des deux années d'expérimentation.

Légende : D1, D2, D3, D4 et D5 représentent les densités de semis à 41667 plants/ha $(0,30 \mathrm{~m} \times 0,80 \mathrm{~m}$ à 1 plant/poquet, 62500 plants/ha $(0,40 \mathrm{~m} \times 0,80 \mathrm{~m}$ à 2 plants/poquet), 62500 plants/ha $(0,20 \mathrm{~m} \times 0,80 \mathrm{~m}$ à 1 plant/poquet), 50000 plants/ha $(0,50 \mathrm{~m} \times 0,80$ $\mathrm{m}$ à 2 plants/poquet) et 41667 plants/ha $(0,60 \mathrm{~m} \times 0,80 \mathrm{~m}$ à 2 plants/poquet), respectivement. Les barres d'erreur représentent l'écart type par rapport à la moyenne.

Tableau 2 : Analyse de la variance des rendements moyens de coton graine en fonction de la période et densité de semis par site et par année d'essai.

\begin{tabular}{|c|c|c|c|c|c|c|c|c|c|}
\hline \multirow{2}{*}{$\begin{array}{l}\text { Sites } \\
\text { Années essai } \\
\end{array}$} & & \multicolumn{4}{|c|}{ Angaradébou } & \multicolumn{2}{|l|}{ Okpara } & \\
\hline & & 2017 & & 2018 & & 2017 & & 2018 & \\
\hline Sources & ddl. & F value & $\operatorname{Pr}(>\mathrm{F})$ & F value & $\operatorname{Pr}(>\mathrm{F})$ & F value & $\operatorname{Pr}(>\mathrm{F})$ & F value & $\operatorname{Pr}(>\mathrm{F})$ \\
\hline Période & 3 & 1,12 & $0,390 \mathrm{~ns}$ & 2,78 & $0,102 \mathrm{~ns}$ & 57,05 & $0,000^{\star \star \star}$ & 98,96 & $0,000^{* \star \star}$ \\
\hline Densité & 4 & 0,71 & $0,591 \mathrm{~ns}$ & 4,29 & $0,005^{\star *}$ & 3,11 & $0,023^{*}$ & 0,39 & $0,812 \mathrm{~ns}$ \\
\hline Période:Densité & 12 & 0,99 & $0,471 \mathrm{~ns}$ & 0,74 & $0,708 \mathrm{~ns}$ & 1,43 & $0,187 \mathrm{~ns}$ & 1,82 & $0,072 \mathrm{~ns}$ \\
\hline Résidus & 48 & & & & & & & & \\
\hline
\end{tabular}

ddl. : degré de liberté. ns : effet non significatif. *, ${ }^{* *}$ et ${ }^{* * *}$ : effet significatif à 0,$05 ; 0,01$ et 0,001 , respectivement.

Tableau 3 : Analyse de la variance des rendements moyens en coton graine en fonction de la période et densité de semis par site pour l'ensemble des deux années d'essai (2017 et 2018).

\begin{tabular}{llllll}
\hline Sites & \multicolumn{3}{l}{ Angaradébou } & & Okpara \\
\hline Sources & ddl. & F value & $\operatorname{Pr}(>\mathrm{F})$ & F value & $\operatorname{Pr}(>\mathrm{F})$ \\
\hline Année & 1 & 5,497 & $0,101 \mathrm{~ns}$ & 10,29 & $0,049^{*}$ \\
Période & 3 & 1,729 & $0,230 \mathrm{~ns}$ & 92,17 & $0,000^{\star * *}$ \\
Densité & 4 & 1,910 & $0,113 \mathrm{~ns}$ & 1,614 & $0,175 \mathrm{~ns}$ \\
Année x Densité & 4 & 2,320 & $0,061 \mathrm{~ns}$ & 1,727 & $0,148 \mathrm{~ns}$ \\
Période:Densité & 12 & 0,358 & $0,975 \mathrm{~ns}$ & 1,412 & $0,169 \mathrm{~ns}$ \\
Résidus & 120 & & & & \\
\hline
\end{tabular}

ddl. : degré de liberté. ns : effet non significatif. ${ }^{*}$ et ${ }^{* * *}$ : effet significatif à 0,05 et 0,001 , respectivement. 
Effet de la période et densité de semis sur la hauteur des plants de cotonnier: A Angaradébou, les plus hauts plants de cotonnier ont été observés sous $\mathrm{P} 2$ (semis au cours de la première décade de juin) à $30 \mathrm{JAL}$ $(37,91 \pm 2,84 \mathrm{~cm})$ et $60 \mathrm{JAL}(107,59 \pm 5,39 \mathrm{~cm})$. Cette tendance a été inversée à $90 \mathrm{JAL}$ où $\mathrm{P} 1$ (semis au cours de la quatrième décade de mai) a enregistré les plus hauts plants $(138,23 \pm 4,19 \mathrm{~cm})$ comparativement aux autres périodes de semis (Tableau 4). Par contre sur le site de Okpara, P3 (21 au 30 juin) a enregistré les plus hauts plants de cotonnier à $60 \mathrm{JAL}(88,97 \pm 10,38 \mathrm{~cm})$ et $90 \mathrm{JAL}(137,08 \pm 9,98 \mathrm{~cm})$ comparativement aux autres périodes (Tableau 4). Dans cette zone, le semis au cours de la première décade de juillet (P4) a réduit le développement végétatif des cotonniers par rapport aux autres périodes. Sur l'ensemble des deux années d'essai, la période de semis a significativement affecté la hauteur des plants de cotonnier aux différentes dates d'observation sur les deux sites $(P<0,05$; Tableau 4) sauf au 30ème jour après levée sur le site de Okpara $(P>$ 0,05 ; Tableau 4). Par rapport à la densité de semis, elle n'a pas affecté la hauteur des plants aux différentes dates d'observation sur les deux sites ayant abrité les essais $(P>0,05$; Tableau 4). L'interaction entre la période et la densité de semis n'a été significative qu'à 90 JAL à Okpara $(P=0,008$; Tableau 4$)$.

Effet de la période et densité de semis sur les paramètres du plant mapping: Les paramètres de plant mapping ont été globalement dans les mêmes proportions pour la période de semis. L'analyse statistique n'a montré aucune différence significative entre les périodes de semis pour l'ensemble des deux années d'essai à Angaradébou pour les différents paramètres de plant mapping considérés (NiPBF, NBV et NBF ; $P>0,05$; Tableau 5). Par rapport à la densité de semis, D2 (41667 plants/ha : 0,60 $\mathrm{m} \times 0,80 \mathrm{~m}$ à 2 plants/poquet) a significativement $(P<0,05$; Tableau $5)$ réduit le nombre de branches végétatives et le niveau d'insertion de la première branche fructifère (NiPBF) comparativement aux autres densités de semis. Par ailleurs, D4 (62500 plants/ha : $0,40 \mathrm{~m} \times 0,80 \mathrm{~m}$ à 2 plants/poquet) a enregistré le nombre de branches fructifères (NBF) le plus élevé relativement aux autres densités de semis (Tableau 5). Sur le site de Okpara, ni la période de semis, ni la densité de semis n'a impacté négativement les paramètres de plant mapping considérés pour l'ensemble des deux années d'essai ( $P$ $>0,05$; Tableau 5). 
Tableau 4 : Hauteur des plants de cotonnier à 30, 60 et 90 jours après levée en fonction de la période et la densité de semis par site pour l'ensemble des deux années d'essai.

\begin{tabular}{|c|c|c|c|c|c|c|c|}
\hline \multirow{2}{*}{ Pér. } & & \multicolumn{3}{|c|}{ Hauteur des cotonniers $(\mathrm{cm})$} & \multirow{2}{*}{\multicolumn{3}{|c|}{ Okpara }} \\
\hline & Dens. & \multicolumn{3}{|c|}{ Angaradébou } & & & \\
\hline & & $30 \mathrm{JAL}$ & $60 \mathrm{JAL}$ & $90 \mathrm{JAL}$ & $30 \mathrm{JAL}$ & $60 \mathrm{JAL}$ & $90 \mathrm{JAL}$ \\
\hline \multirow[t]{5}{*}{ P1 } & D1 & $35,50 \pm 3,87$ & $98,43 \pm 5,73$ & $138,84 \pm 4,43$ & $24,21 \pm 5,43$ & $83,80 \pm 7,35$ & $125,05 \pm 8,58$ \\
\hline & D2 & $35,99 \pm 3,15$ & $99,09 \pm 5,07$ & $138,35 \pm 3,92$ & $25,98 \pm 6,00$ & $84,68 \pm 5,76$ & $125,98 \pm 6,31$ \\
\hline & D3 & $36,58 \pm 3,74$ & $99,95 \pm 4,00$ & $137,76 \pm 4,99$ & $25,64 \pm 8,63$ & $81,26 \pm 8,34$ & $116,68 \pm 14,95$ \\
\hline & D4 & $35,79 \pm 3,24$ & $99,51 \pm 7,38$ & $136,51 \pm 4,35$ & $26,04 \pm 6,90$ & $82,84 \pm 11,30$ & $134,05 \pm 11,15$ \\
\hline & D5 & $34,56 \pm 3,33$ & $98,24 \pm 7,08$ & $139,66 \pm 3,57$ & $25,66 \pm 4,65$ & $83,29 \pm 12,22$ & $125,33 \pm 12,91$ \\
\hline \multicolumn{2}{|c|}{ Moyenne P1 } & $35.68 \pm 3.36 \mathrm{~B}$ & $99,04 \pm 5,71 \mathrm{C}$ & $138,23 \pm 4,19 \mathrm{~A}$ & $25,51 \pm 6,16$ & $83,17 \pm 83,17 \mathrm{~B}$ & $125,42 \pm 125,42 \mathrm{C}$ \\
\hline \multirow[t]{5}{*}{ P2 } & D1 & $37,78 \pm 3,47$ & $106,08 \pm 3,91$ & $132,74 \pm 6,49$ & $23,55 \pm 2,30$ & $88,88 \pm 7,17$ & $135,58 \pm 10,38$ \\
\hline & D2 & $38,18 \pm 1,75$ & $107,90 \pm 5,75$ & $133,60 \pm 6,11$ & $24,40 \pm 2,57$ & $84,05 \pm 7,21$ & $122,26 \pm 13,83$ \\
\hline & D3 & $37,74 \pm 2,71$ & $105,67 \pm 4,93$ & $130,61 \pm 6,50$ & $24,60 \pm 2,41$ & $89,60 \pm 15,17$ & $132,75 \pm 9,73$ \\
\hline & D4 & $37,26 \pm 3,79$ & $107,40 \pm 5,87$ & $132,41 \pm 9,41$ & $24,81 \pm 2,26$ & $88,40 \pm 4,42$ & $131,35 \pm 12,16$ \\
\hline & D5 & $38,58 \pm 2,66$ & $110,93 \pm 5,89$ & $136,56 \pm 1,99$ & $23,59 \pm 2,44$ & $88,99 \pm 8,64$ & $133,21 \pm 6,57$ \\
\hline \multicolumn{2}{|c|}{ Moyenne P2 } & $37,78 \pm 2.84 \mathrm{~A}$ & $107,59 \pm 5,3 \mathrm{~A}$ & $133,19 \pm 6,51 \mathrm{~B}$ & $24,19 \pm 2,34$ & $87,98 \pm 87,98 \mathrm{~A}$ & $131,03 \pm 131,03 B$ \\
\hline \multirow[t]{5}{*}{ P3 } & $\mathrm{D} 1$ & $35,31 \pm 5,96$ & $103,30 \pm 4,51$ & $135,39 \pm 4,53$ & $25,58 \pm 2,90$ & $86,60 \pm 8,60$ & $133,78 \pm 10,34$ \\
\hline & D2 & $35,40 \pm 5,83$ & $103,85 \pm 3,70$ & $132,25 \pm 3,98$ & $25,64 \pm 3,25$ & $94,38 \pm 10,85$ & $138,56 \pm 9,63$ \\
\hline & D3 & $36,09 \pm 6,40$ & $102,85 \pm 5,57$ & $132,73 \pm 6,89$ & $26,84 \pm 0,98$ & $93,25 \pm 9,58$ & $137,86 \pm 6,98$ \\
\hline & D4 & $36,08 \pm 6,14$ & $104,73 \pm 4,61$ & $135,93 \pm 4,95$ & $25,05 \pm 2,13$ & $83,90 \pm 6,20$ & $133,49 \pm 9,49$ \\
\hline & D5 & $36,43 \pm 5,77$ & $102,69 \pm 4,23$ & $132,53 \pm 2,52$ & $25,39 \pm 5,05$ & $86,70 \pm 13,52$ & $141,71 \pm 12,69$ \\
\hline \multicolumn{2}{|c|}{ Moyenne P3 } & $35.86 \pm 5.72 B$ & $103,48 \pm 4,3 \mathrm{~B}$ & $133,77 \pm 4,80 \mathrm{~B}$ & $25,70 \pm 3,06$ & $88,97 \pm 88,97 \mathrm{~A}$ & $137,08 \pm 137,08 \mathrm{~A}$ \\
\hline \multirow[t]{5}{*}{$\mathrm{P} 4$} & $\mathrm{D} 1$ & $33,60 \pm 3,84$ & $106,18 \pm 5,68$ & $133,61 \pm 3,76$ & $25,81 \pm 10,2$ & $64,19 \pm 21,14$ & $107,88 \pm 12,70$ \\
\hline & D2 & $33,80 \pm 3,67$ & $105,14 \pm 4,38$ & $133,08 \pm 2,89$ & $25,21 \pm 11,64$ & $65,49 \pm 28,61$ & $101,29 \pm 12,82$ \\
\hline & D3 & $33,21 \pm 2,79$ & $106,65 \pm 6,64$ & $135,13 \pm 4,94$ & $26,83 \pm 9,18$ & $72,56 \pm 20,69$ & $113,39 \pm 8,35$ \\
\hline & D4 & $33,35 \pm 3,36$ & $106,45 \pm 6,32$ & $134,81 \pm 4,19$ & $19,16 \pm 9,50$ & $59,36 \pm 20,52$ & $114,70 \pm 14,60$ \\
\hline & D5 & $33,84 \pm 3,09$ & $106,51 \pm 5,59$ & $134,33 \pm 4,29$ & $22,26 \pm 6,79$ & $58,31 \pm 16,57$ & $107,50 \pm 5,40$ \\
\hline \multicolumn{2}{|c|}{ Moyenne P4 } & $33,56 \pm 3.20 \mathrm{C}$ & $106,19 \pm 5,5 \mathrm{~A}$ & $134,19 \pm 15,33 \mathrm{~B}$ & $23,86 \pm 9,56$ & $63,98 \pm 63,98 \mathrm{C}$ & $108,95 \pm 108,95 \mathrm{D}$ \\
\hline \multirow{5}{*}{ 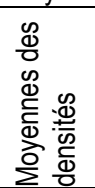 } & $\mathrm{D} 1$ & $35,55 \pm 4,44$ & $103,49 \pm 5,74$ & $135,14 \pm 5,24$ & $24,79 \pm 5,93$ & $80,87 \pm 15,51$ & $125,57 \pm 15,02$ \\
\hline & D2 & $35,84 \pm 4,02$ & $103,99 \pm 5,59$ & $134,32 \pm 4,82$ & $25,31 \pm 6,56$ & $82,15 \pm 18,53$ & $122,02 \pm 17,20$ \\
\hline & D3 & $35,90 \pm 4,32$ & $103,78 \pm 5,75$ & $134,06 \pm 6,22$ & $25,98 \pm 6,18$ & $84,17 \pm 15,84$ & $125,17 \pm 14,47$ \\
\hline & D4 & $35,62 \pm 4,33$ & $104,52 \pm 6,59$ & $134,92 \pm 6,03$ & $23,77 \pm 6,39$ & $78,63 \pm 16,41$ & $128,40 \pm 13,98$ \\
\hline & D5 & $35,85 \pm 4,15$ & $104,59 \pm 7,28$ & $135,77 \pm 4,08$ & $24,23 \pm 4,94$ & $79,32 \pm 17,61$ & $126,94 \pm 15,97$ \\
\hline \multicolumn{2}{|c|}{ Prob.période } & $0,006^{\star *}$ & $0,000^{\star \star *}$ & $0,001^{* *}$ & $0,382 \mathrm{~ns}$ & $0,000^{\star \star *}$ & $0,000^{\star \star *}$ \\
\hline \multicolumn{2}{|c|}{ Prob.densité } & $0,728 \mathrm{~ns}$ & $0,540 \mathrm{~ns}$ & $0,345 \mathrm{~ns}$ & $0,181 \mathrm{~ns}$ & $0,151 \mathrm{~ns}$ & $0,084 \mathrm{~ns}$ \\
\hline \multicolumn{2}{|c|}{ Prob.Pér.xDens. } & $0,096 \mathrm{~ns}$ & $0,174 n s$ & $0,104 n s$ & $0,177 \mathrm{~ns}$ & $0,315 \mathrm{~ns}$ & 0,008 ** \\
\hline
\end{tabular}

Légende $:$ Pér. $=$ Période, Dens. $=$ Densité et Prob. = Probabilité. A Angaradébou, P1, P2, P3 et P4 représentent les périodes de semis entre 20-30 mai, 1-10 juin, 11-20 juin et 21-30 juin (2017 et 2018), respectivement, tandis qu'à Okpara $P 1, P 2, P 3$ et $P 4$ représentent les périodes de semis entre 1-10 juin, 11-20 juin, 21-30 juin et 1-10 juillet (2017 et 2018), respectivement.

D1, D2, D3, D4 et D5 représentent les densités de semis à 41667 plants/ha $(0,30 \mathrm{~m} \times 0,80 \mathrm{~m}$ à 1 plant/poquet, 62500 plants/ha $(0,40 \mathrm{~m} \times 0,80 \mathrm{~m}$ à 2 plants/poquet $), 62500$ plants/ha $(0,20 \mathrm{~m} \times 0,80 \mathrm{~m}$ à 1 plant/poquet), 50000 plants/ha (0,50 m x 0,80 m à 2 plants/poquet) et 41667 plants/ha (0,60 $\mathrm{m} \mathrm{x} \mathrm{0,80} \mathrm{m} \mathrm{à} 2$ plants/poquet), respectivement dans les différentes zones. ns : effet non significatif. ** et ${ }^{* * *}$ : effet significatif à 0,01 et 0,001 , respectivement.

Les moyennes suivies des mêmes lettres et de même caractère dans une même colonne ne sont pas significativement différentes au seuil de $5 \%$. 
Tableau 5 : Plant mapping en fonction des périodes et densités de semis à Angaradébou et Okpara.

\begin{tabular}{|c|c|c|c|c|c|c|c|}
\hline \multirow[t]{2}{*}{ Pér, } & Dens & & Angaradébou & & Okpara & & \\
\hline & & NiPBF & NBV & NBF & NiPBF & NBV & NBF \\
\hline \multirow[t]{5}{*}{ P1 } & D1 & $6,60 \pm 0,43$ & $1,78 \pm 0,37$ & $19,01 \pm 0,83$ & $6,64 \pm 1,10$ & $2,21 \pm 0,42$ & $14,15 \pm 1,67$ \\
\hline & D2 & $6,32 \pm 0,33$ & $1,53 \pm 0,37$ & $18,88 \pm 1,07$ & $7,09 \pm 1,13$ & $2,16 \pm 0,32$ & $14,26 \pm 2,17$ \\
\hline & D3 & $6,51 \pm 0,52$ & $1,40 \pm 0,24$ & $18,63 \pm 1,11$ & $7,14 \pm 1,52$ & $2,16 \pm 0,44$ & $13,93 \pm 3,04$ \\
\hline & D4 & $6,55 \pm 0,33$ & $2,00 \pm 0,49$ & $19,23 \pm 1,01$ & $6,51 \pm 1,14$ & $3,48 \pm 1,96$ & $15,49 \pm 1,56$ \\
\hline & D5 & $6,43 \pm 0,36$ & $1,76 \pm 0,54$ & $19,25 \pm 1,13$ & $6,69 \pm 1,47$ & $2,31 \pm 0,47$ & $14,98 \pm 3,20$ \\
\hline \multicolumn{2}{|c|}{ Moyenne P1 } & $6,48 \pm 0,31$ & $1,69 \pm 0,28$ & $19,00 \pm 1,01$ & $6,81 \pm 1,25$ & $2,47 \pm 1,04$ & $14,56 \pm 2,37$ \\
\hline \multirow[t]{5}{*}{ P2 } & D1 & $6,48 \pm 0,35$ & $1,65 \pm 0,27$ & $18,40 \pm 1,41$ & $6,60 \pm 1,04$ & $2,78 \pm 0,68$ & $14,93 \pm 1,77$ \\
\hline & D2 & $6,34 \pm 0,24$ & $1,84 \pm 0,39$ & $19,30 \pm 1,79$ & $6,76 \pm 1,03$ & $2,43 \pm 0,58$ & $13,86 \pm 2,93$ \\
\hline & D3 & $6,38 \pm 0,35$ & $1,53 \pm 0,27$ & $17,80 \pm 1,03$ & $6,44 \pm 0,99$ & $2,40 \pm 0,41$ & $14,21 \pm 3,49$ \\
\hline & D4 & $6,41 \pm 0,16$ & $1,84 \pm 0,51$ & $19,15 \pm 1,90$ & $6,4 \pm 1,45$ & $4,78 \pm 5,77$ & $15,26 \pm 2,38$ \\
\hline & D5 & $6,61 \pm 0,43$ & $2,13 \pm 0,69$ & $19,53 \pm 1,25$ & $6,80 \pm 1,32$ & $2,24 \pm 0,50$ & $14,73 \pm 2,47$ \\
\hline \multicolumn{2}{|c|}{ Moyenne P2 } & $6,44 \pm 0,32$ & $1,80 \pm 0,48$ & $18,84 \pm 1,57$ & $6,62 \pm 1,13$ & $2,92 \pm 2,67$ & $14,60 \pm 2,58$ \\
\hline \multirow[t]{5}{*}{ P3 } & D1 & $6,51 \pm 0,27$ & $1,85 \pm 0,08$ & $19,05 \pm 0,86$ & $7,01 \pm 0,82$ & $2,61 \pm 0,43$ & $14,51 \pm 0,59$ \\
\hline & D2 & $6,38 \pm 0,21$ & $1,59 \pm 0,22$ & $18,43 \pm 1,11$ & $6,73 \pm 0,85$ & $2,36 \pm 0,50$ & $14,43 \pm 2,25$ \\
\hline & D3 & $6,31 \pm 0,15$ & $1,64 \pm 0,15$ & $18,34 \pm 1,27$ & $6,94 \pm 0,98$ & $4,98 \pm 7,30$ & $14,84 \pm 1,72$ \\
\hline & D4 & $6,49 \pm 0,17$ & $1,90 \pm 0,26$ & $19,25 \pm 1,18$ & $6,81 \pm 1,17$ & $2,50 \pm 0,33$ & $14,74 \pm 0,91$ \\
\hline & D5 & $6,48 \pm 0,19$ & $1,89 \pm 0,24$ & $18,14 \pm 0,91$ & $8,61 \pm 5,47$ & $2,36 \pm 0,45$ & $15,24 \pm 1,95$ \\
\hline \multicolumn{2}{|c|}{ Moyenne P3 } & $6,43 \pm 0,21$ & $1,77 \pm 0,23$ & $18,64 \pm 1,11$ & $7,22 \pm 2,56$ & $2,96 \pm 3,28$ & $14,75 \pm 1,56$ \\
\hline \multirow[t]{5}{*}{ P4 } & D1 & $6,43 \pm 0,18$ & $1,98 \pm 0,17$ & $18,76 \pm 1,02$ & $6,40 \pm 1,35$ & $2,23 \pm 0,34$ & $13,51 \pm 1,26$ \\
\hline & D2 & $6,60 \pm 0,43$ & $1,65 \pm 0,15$ & $18,06 \pm 0,31$ & $6,35 \pm 1,22$ & $1,83 \pm 0,59$ & $12,90 \pm 0,91$ \\
\hline & D3 & $6,55 \pm 0,28$ & $1,65 \pm 0,30$ & $18,51 \pm 0,87$ & $6,69 \pm 1,34$ & $2,34 \pm 0,54$ & $13,48 \pm 1,91$ \\
\hline & D4 & $6,45 \pm 0,23$ & $1,91 \pm 0,24$ & $18,94 \pm 1,06$ & $6,48 \pm 1,06$ & $2,10 \pm 0,60$ & $13,90 \pm 2,04$ \\
\hline & D5 & $6,53 \pm 0,38$ & $2,09 \pm 0,24$ & $18,66 \pm 1,00$ & $6,73 \pm 1,23$ & $1,91 \pm 0,36$ & $13,43 \pm 1,64$ \\
\hline \multicolumn{2}{|c|}{ Moyenne P4 } & $6,51 \pm 0,31$ & $1,86 \pm 0,28$ & $18,59 \pm 0,90$ & $6,53 \pm 1,19$ & $2,08 \pm 0,51$ & $13,44 \pm 1,56$ \\
\hline \multirow{5}{*}{ 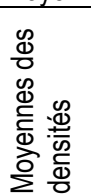 } & D1 & $6,50 \pm 0,31 a b$ & $1,81 \pm 0,26 b$ & $18,81 \pm 1,04 a b$ & $6,66 \pm 1,06$ & $2,46 \pm 0,52$ & $14,28 \pm 1,43$ \\
\hline & D2 & $6,41 \pm 0,32 \mathrm{c}$ & $1,65 \pm 0,31 \mathrm{c}$ & $18,67 \pm 1,23 b c$ & $6,73 \pm 1,05$ & $2,19 \pm 0,54$ & $13,86 \pm 2,17$ \\
\hline & D3 & $6,44 \pm 0,35 \mathrm{bc}$ & $1,55 \pm 0,26 c$ & $18,32 \pm 1,07 c$ & $6,80 \pm 1,20$ & $2,97 \pm 3,69$ & $14,11 \pm 2,56$ \\
\hline & D4 & $6,48 \pm 0,23 a b c$ & $1,91 \pm 0,38 a b$ & $19,14 \pm 1,27 a$ & $6,57 \pm 1,17$ & $3,21 \pm 3,10$ & $14,85 \pm 1,83$ \\
\hline & D5 & $6,51 \pm 0,34 a$ & $1,97 \pm 0,47 a$ & $18,90 \pm 1,16 \mathrm{ab}$ & $7,21 \pm 2,94$ & $2,21 \pm 0,46$ & $14,59 \pm 2,38$ \\
\hline \multicolumn{2}{|c|}{ Prob,Pér, } & $0,051 \mathrm{~ns}$ & $0,126 \mathrm{~ns}$ & $0,328 \mathrm{~ns}$ & $0,587 \mathrm{~ns}$ & $0,312 \mathrm{~ns}$ & $0,127 \mathrm{~ns}$ \\
\hline \multicolumn{2}{|c|}{ Prob,Dens, } & $0,003^{\star \star}$ & $0,000^{\star * *}$ & $0,000^{* * *}$ & $0,277 n s$ & $0,236 \mathrm{~ns}$ & $0,098 \mathrm{~ns}$ \\
\hline \multicolumn{2}{|c|}{ Prob, Pér,xDens, } & $0,000^{* * *}$ & $0,000^{* * *}$ & $0,000^{* * *}$ & $0,522 \mathrm{~ns}$ & $0,405 n s$ & $0,950 \mathrm{~ns}$ \\
\hline
\end{tabular}

Légende : NiPBF : Niveau d'insertion de la première branche fructifère, NBV : Nombre de branches végétatives, NBF : Nombre de branches fructifères. Pér. = Période, Dens. = Densité et Prob. = Probabilité.

A Angaradébou, P1, P2, P3 et P4 représentent les périodes de semis entre 20-30 mai, 1-10 juin, 11-20 juin et 21-30 juin (2017 et 2018), respectivement, tandis qu'à Okpara P1, P2, P3 et P4 représentent les périodes de semis entre 1-10 juin, 11-20 juin, 21-30 juin et 1-10 juillet (2017 et 2018), respectivement.

D1, D2, D3, D4 et D5 représentent les densités de semis à 41667 plants/ha $(0,30 \mathrm{~m} \times 0,80 \mathrm{~m}$ à 1 plant/poquet, 62500 plants/ha $(0,40 \mathrm{~m} \times 0,80 \mathrm{~m}$ à 2 plants/poquet), 62500 plants/ha (0,20 $\mathrm{m} \times 0,80$ $\mathrm{m}$ à 1 plant/poquet), 50000 plants/ha $(0,50 \mathrm{~m} \times 0,80 \mathrm{~m}$ à 2 plants/poquet) et 41667 plants/ha ( $0,60 \mathrm{~m} \times 0,80 \mathrm{~m}$ à 2 plants/poquet), respectivement dans les différentes zones. ns : effet non significatif. ${ }^{* *}$ et ${ }^{* * *}$ : effet significatif à 0,01 et 0,001 , respectivement.

Les moyennes suivies des mêmes lettres et de même caractère dans une même colonne ne sont pas significativement différentes au seuil de $5 \%$. 
Effet de la période et de la densité de semis sur le shedding des organes de reproduction : Le shedding physiologique des organes de reproduction a été important à Angaradébou où il a été observé des quantités importantes de chute de boutons floraux sains et de capsules saines relativement à Okpara (Tableaux 6 et 7). Pour l'ensemble des deux années d'expérimentation à Angaradébou, l'analyse statistique a révélé de différence significative entre les périodes de semis pour le nombre de boutons floraux sains (BFS) et de boutons floraux parasités (BFP ; $P<0,05$; Tableau $6)$. Cependant, aucune différence significative n'a été observée entre les périodes de semis pour le nombre de capsules saines et parasitées $(P>0,05$; Tableau 6$)$. La densité de semis a significativement affecté les paramètres de shedding étudiés $(P<0,05$; Tableau 6$)$ sauf le nombre de boutons floraux parasitées $(P>0,05$; Tableau 6). La densité de semis D3 (62500 plants/ha: $0,20 \mathrm{~m} \times 0,80 \mathrm{~m}$ à 1 plant/poquet) a significativement influencé la chute de boutons floraux sains et de capsules saines par rapport aux autres densités de semis (Tableau 6). Les résultats ont montré de différence significative entre les périodes de semis pour tous les paramètres étudiés $(P<0,05$; Tableau 7$)$, sauf les capsules saines (CapsS) sur le site de Okpara. De même, une différence significative a été observée entre les densités de semis pour BFS, BFP et CapsS $(P<$ 0,05 ; Tableau 7), contrairement au nombre de capsules parasitées $(P>0,05$; Tableau 7). Par ailleurs, les résultats n'ont montré aucune interaction significative entre la période et la densité de semis pour les paramètres de shedding étudiés (Tableau 7). 
Tableau 6 : Shedding des organes de reproduction en fonction des périodes et densités de semis à Angaradébou.

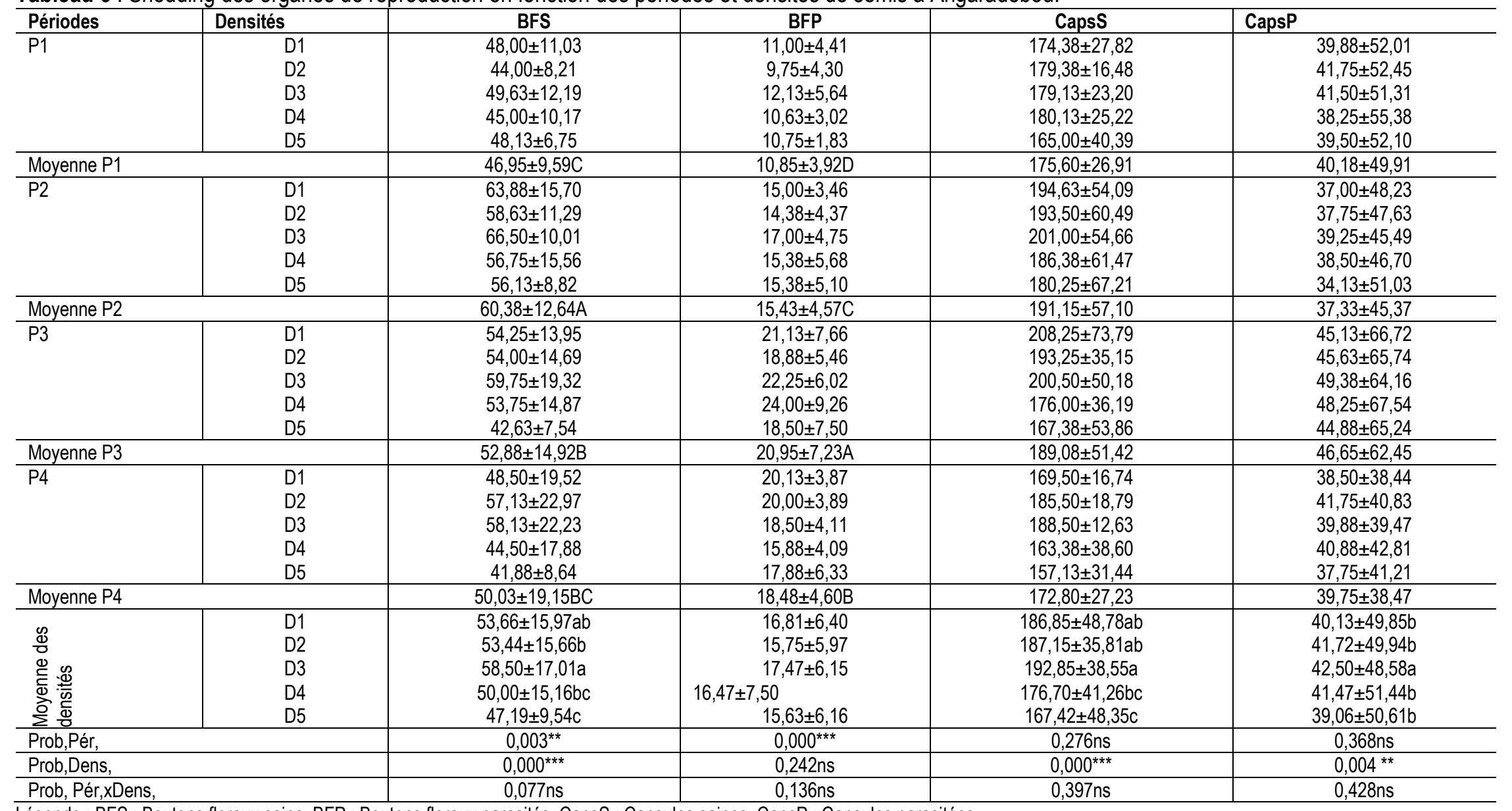

Légende : BFS : Boutons floraux sains, BFP : Boutons floraux parasités, CapsS : Capsules saines, CapsP : Capsules parasitées.

P1, P2, P3 et P4 représentent les périodes de semis entre 20-30 mai, 1-10 juin, 11-20 juin et 21-30 juin (2017 et 2018), respectivement.

D1, D2, D3, D4 et D5 représentent les densités de semis à 41667 plants/ha ( $0,30 \mathrm{~m} \times 0,80 \mathrm{~m}$ à 1 plant/poquet, 62500 plants/ha $(0,40 \mathrm{~m} \times 0,80 \mathrm{~m}$ à 2 plants/poquet), 62500 plants/ha ( $0,20 \mathrm{~m} \times 0,80 \mathrm{~m}$ à 1 plant/poquet), 50000 plants/ha ( $0,50 \mathrm{~m} \times 0,80 \mathrm{~m}$ à 2 plants/poquet) et 41667 plants/ha $(0,60 \mathrm{~m} \times 0,80 \mathrm{~m}$ à 2 plants/poquet), respectivement dans les différentes zones. ns : effet non significatif.Les moyennes suivies des mêmes lettres de même caractère dans une même colonne ne sont pas significativement différentes au seuil de $5 \%$. 
Tableau 7 : Shedding des organes de reproduction en fonction des périodes et densités de semis à Okpara.

\begin{tabular}{|c|c|c|c|c|c|}
\hline Périodes & Densités & BFS & BFP & CapsS & CapsP \\
\hline \multirow[t]{5}{*}{$\mathrm{P} 1$} & D1 & $15,50 \pm 7,72$ & $23,50 \pm 2,89$ & $28,25 \pm 8,66$ & $9,50 \pm 3,42$ \\
\hline & D2 & $15,00 \pm 3,51$ & $26,25 \pm 10,44$ & $29,25 \pm 11,41$ & $12,25 \pm 3,20$ \\
\hline & D3 & $18,52 \pm 8,46$ & $21,75 \pm 6,18$ & $26,75 \pm 6,50$ & $10,50 \pm 6,81$ \\
\hline & D4 & $12,00 \pm 2,45$ & $20,00 \pm 8,12$ & $23,25 \pm 10,81$ & $7,75 \pm 7,85$ \\
\hline & D5 & $9,50 \pm 2,38$ & $21,50 \pm 4,04$ & $19,50 \pm 8,35$ & $9,25 \pm 3,40$ \\
\hline \multicolumn{2}{|c|}{ Moyenne P1 } & $14,52 \pm 5,86 \mathrm{~B}$ & $22,60 \pm 6,51 \mathrm{~B}$ & $25,40 \pm 9,06$ & $9,85 \pm 4,97 \mathrm{~A}$ \\
\hline \multirow[t]{5}{*}{ P2 } & $\mathrm{D} 1$ & $15,00 \pm 3,32$ & $32,75 \pm 10,05$ & $25,50 \pm 7,33$ & $12,00 \pm 6,93$ \\
\hline & D2 & $17,00 \pm 5,00$ & $33,00 \pm 9,83$ & $26,00 \pm 9,06$ & $8,25 \pm 6,08$ \\
\hline & D3 & $22,55 \pm 7,93$ & $29,00 \pm 4,32$ & $28,25 \pm 6,90$ & $10,25 \pm 4,35$ \\
\hline & D4 & $16,53 \pm 8,34$ & $31,00 \pm 11,86$ & $20,00 \pm 9,06$ & $5,00 \pm 3,74$ \\
\hline & D5 & $16,00 \pm 2,45$ & $36,75 \pm 4,99$ & $19,00 \pm 7,53$ & $9,50 \pm 4,20$ \\
\hline \multicolumn{2}{|c|}{ Moyenne P2 } & $17,00 \pm 5,81 \mathrm{~A}$ & $32,50 \pm 8,20 \mathrm{~A}$ & $23,75 \pm 8,03$ & $9,00 \pm 5,21 \mathrm{~A}$ \\
\hline \multirow[t]{5}{*}{ P3 } & D1 & $14,54 \pm 4,86$ & $9,50 \pm 6,35$ & $23,50 \pm 13,30$ & $9,75 \pm 11,18$ \\
\hline & $\mathrm{D} 2$ & $15,53 \pm 7,09$ & $14,25 \pm 1,89$ & $14,00 \pm 6,16$ & $9,00 \pm 1,63$ \\
\hline & D3 & $16,55 \pm 7,04$ & $13,50 \pm 3,70$ & $26,00 \pm 4,24$ & $11,50 \pm 6,03$ \\
\hline & D4 & $10,00 \pm 4,24$ & $8,00 \pm 3,27$ & $17,00 \pm 18,71$ & $3,50 \pm 1,73$ \\
\hline & D5 & $10,00 \pm 2,31$ & $11,25 \pm 10,56$ & $17,00 \pm 7,07$ & $7,50 \pm 3,79$ \\
\hline \multicolumn{2}{|c|}{ Moyenne P3 } & $13,54 \pm 5,53 \mathrm{~B}$ & $11,30 \pm 5,85 \mathrm{C}$ & $19,50 \pm 11,01$ & $8,25 \pm 6,03 \mathrm{~A}$ \\
\hline \multirow[t]{5}{*}{ P4 } & D1 & $6,00 \pm 2,71$ & $7,75 \pm 4,27$ & $18,25 \pm 7,41$ & $5,25 \pm 2,36$ \\
\hline & $\mathrm{D} 2$ & $10,00 \pm 2,38$ & $13,25 \pm 6,40$ & $16,75 \pm 6,80$ & $4,50 \pm 2,65$ \\
\hline & D3 & $13,00 \pm 3,70$ & $7,50 \pm 1,00$ & $25,00 \pm 4,24$ & $2,50 \pm 2,08$ \\
\hline & D4 & $6,00 \pm 2,58$ & $9,75 \pm 3,30$ & $8,25 \pm 6,08$ & $2,25 \pm 0,96$ \\
\hline & D5 & $9,25 \pm 5,19$ & $5,25 \pm 4,92$ & $14,25 \pm 9,71$ & $3,25 \pm 2,06$ \\
\hline \multicolumn{2}{|c|}{ Moyenne P4 } & $9,05 \pm 4,25 \mathrm{C}$ & $8,70 \pm 4,76 \mathrm{C}$ & $16,50 \pm 8,41$ & $3,55 \pm 2,21 \mathrm{~B}$ \\
\hline \multirow{5}{*}{ 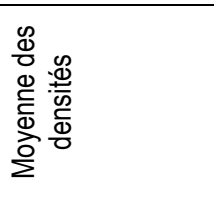 } & D1 & $12,81 \pm 6,09 b c$ & $18,38 \pm 12,12 b$ & $23,88 \pm 9,29 a$ & $9,13 \pm 6,66$ \\
\hline & D2 & $14,69 \pm 5,07 a b$ & $21,69 \pm 11,11 a$ & $21,50 \pm 10,09 a b$ & $8,50 \pm 4,41$ \\
\hline & D3 & $17,56 \pm 7,10 a$ & $17,94 \pm 9,23 b$ & $26,50 \pm 5,16 a$ & $8,69 \pm 5,92$ \\
\hline & D4 & $11,19 \pm 6,00 \mathrm{c}$ & $17,19 \pm 11,66 b$ & $17,13 \pm 12,26 b$ & $4,63 \pm 4,51$ \\
\hline & D5 & $11,19 \pm 4,13 \mathrm{c}$ & $18,69 \pm 13,69 b$ & $17,44 \pm 7,66 b$ & $7,38 \pm 4,03$ \\
\hline \multicolumn{2}{|c|}{ Prob,Pér, } & $0,003^{* *}$ & $0,003^{* *}$ & $0,241 \mathrm{~ns}$ & $0,035^{*}$ \\
\hline \multicolumn{2}{|c|}{ Prob,Dens, } & $0,002^{* *}$ & $0,002^{* *}$ & $0,003^{\star *}$ & $0,058 \mathrm{~ns}$ \\
\hline \multicolumn{2}{|c|}{ Prob, Pér,xDens, } & $0,905 n s$ & $0,905 n s$ & $0,776 \mathrm{~ns}$ & $0,935 n s$ \\
\hline
\end{tabular}

Légende : BFS : Boutons floraux sains, BFP : Boutons floraux parasités, CapsS : Capsules saines, CapsP : Capsules parasitées.

$P 1, P 2, P 3$ et $P 4$ représentent les périodes de semis entre 1-10 juin, 11-20 juin, 21-30 juin et 1-10 juillet (2017 et 2018), respectivement.

D1, D2, D3, D4 et D5 représentent les densités de semis à 41667 plants/ha $(0,30 \mathrm{~m} \times 0,80 \mathrm{~m}$ à 1 plant/poquet, 62500 plants/ha $(0,40 \mathrm{~m} \times 0,80 \mathrm{~m}$ à 2 plants/poquet), 62500 plants/ha $(0,20 \mathrm{~m} \times 0,80 \mathrm{~m}$ à 1 plant/poquet), 50000 plants/ha $(0,50 \mathrm{~m} \times 0,80 \mathrm{~m}$ à 2 plants/poquet) et 41667 plants/ha $(0,60 \mathrm{~m} \times 0,80 \mathrm{~m}$ à 2 plants/poquet), respectivement dans les différentes zones. ns : effet non significatif.

Les moyennes suivies des mêmes lettres de même caractère dans une même colonne ne sont pas significativement différentes au seuil de $5 \%$. 


\section{DISCUSSION}

Effet de la période et la densité de semis sur le rendement en coton graine: Les résultats de la présente étude ont montré une influence de la période de semis sur le rendement en coton graine. Cet effet a été significatif à Okpara pour l'ensemble des deux années. A Angaradébou qui représente la zone nord, les quatre décades de semis testées (20-30 mai ; 1-10 juin ; 11-20 juin et 21-30 juin) ont généré des rendements en coton graine équivalents durant les deux années d'expérimentation. Ce résultat montre un étalement $d u$ semis dans cette zone où le cotonnier est capable de rattraper un écart de près de trois décades. La recommandation actuelle de semis s'étend du 20 mai au 20 juin. A travers les résultats obtenus, cette recommandation reste d'actualité. Mais on peut étendre la période optimale de semis jusqu'au 30 juin, étant donné que les résultats ont été similaires pour les quatre décades de semis durant les deux années d'expérimentation (Figures 5 \& 6). Si les rendements des deux premières décades n'ont pas été significativement meilleurs, cela pourrait être expliqué par la prévalence des conditions pluviométriques défavorables au démarrage des semis. De plus, les quatre décades de semis auraient bénéficié de la même façon du pic de minéralisation de la matière organique du sol. Ce qui aurait mis les cotonniers dans les mêmes conditions du point de vue de disponibilité de nutriments du sol. Des résultats similaires ont été obtenus sur la culture du soja par De Bruin \& Pedersen (2008). En effet, ces derniers ont obtenu des effets non significatifs de quatre périodes de semis sur le rendement du soja dans l'lowa, aux Etats-Unis d'Amérique. Par contre à Okpara, qui représente le centre-nord du pays, les rendements n'ont pas été stables sur les deux ans. Si en 2017, la première décade de juin (1-10 juin) a donné le meilleur rendement, mais significativement équivalent au rendement obtenu durant la deuxième décade de juin (10-20 juin), la tendance a été inversée en 2018. De plus, le meilleur rendement moyen pour l'ensemble des deux ans est au profit de la deuxième décade de juin (Figures $3 \& 4$ ). La recommandation de semis en vigueur dans cette zone s'étend du 10 juin au 5 juillet. A travers les résultats obtenus, cette recommandation reste d'actualité. Mais tout semis dans la première décade de juillet serait déconseillé, car durant les deux ans, cette dernière décade a généré les plus faibles rendements comparativement aux autres périodes. Ainsi, dans le centre-nord, il n'est pas conseillé de faire des semis précoces, ni de semis tardifs. La période optimale de semis s'étale donc seulement sur les deux dernières décades du mois de juin, contrairement à la zone nord où les semis peuvent être étalés sur quatre décades (20 mai au 30 juin). Par ailleurs, à l'étape actuelle où la recherche n'a pas recommandé des variétés précoces pour les éventuels semis tardifs, ces résultats montrent qu'il n'est pas utile de faire des recommandations de semis du coton durant le mois de juillet pour l'ensemble de la zone nord et du centre-nord. Ces résultats corroborent ceux de Arshad et al. (2007), Bozbek et al. (2006), Awan et al. (2011) qui ont observé de réduction substantielle de rendement de coton graine due à un semis tardif au Pakistan. Similairement, Sêkloka et al. (2015) ont rapporté une réduction de rendement de 12\% pour des variétés exotiques précoces et $30 \%$ pour des variétés tardives due à des semis tardifs au nord et au sud Bénin. En effet, les semis tardifs sont souvent confrontés à des conditions environnementales préjudiciables (excès de température, pluies abondantes ou déficitaires) au développement et au maintien des organes fructifères. Ce qui affecte négativement le rendement final (Ali et al., 2004; Bilal et al., 2015). Une réduction de rendement en coton graine de $34,13 \%$, due à un retard de deux semaines dans le semis du cotonnier par rapport à la date de semis recommandée a été rapportée au Pakistan (Bozbek et al., 2006). En ce qui concerne la densité de semis, si en 2017 un effet positif a été observé sur le rendement à Okpara, la tendance a été inversée en 2018 où cet effet significatif a été observé à Angaradébou. Sur les deux sites ayant abrité les essais, le meilleur rendement a été obtenu avec la densité D3 (62500 plants/ha: $0,80 \mathrm{~m} \times 0,2 \mathrm{~m} ; 1$ plant/poquet). De plus, l'instabilité de l'expression des différentes densités peut être expliquée par la variation des conditions climatiques en fonction de l'année. Du point de vue de nombre de plants à l'hectare, la densité D3 est égale à D2 ; cependant, le nombre de plants par poquet et la distance inter-poquets ont varié. Les poquets de la densité $\mathrm{D} 2$ ont été démariés à deux plants avec des écartements de $0,4 \mathrm{~m}$ entre poquets ; alors que la distance inter-poquets de D3 était 0,20 m, et chaque poquet a été démarié à 1 plant. Ainsi, la différence de rendement aurait résulté de la différence de microclimat généré par la géométrie de semis pour chacune des densités. En dehors de D3, les autres densités s'équivalent. Ces résultats sont conformes à ceux de Yang et al. (2014). En effet, pour ces derniers, une densité de semis optimale du cotonnier crée un microenvironnement bénéfique à travers le couvert végétal pour la croissance et le développement des plantes, ce qui améliore le rendement. La géométrie de 
semis de la densité D3 est favorable à la pénétration des rayons solaires à l'intérieur des plants, ce qui permet d'optimiser la synthèse des assimilas à travers la photosynthèse pour la croissance végétative et la formation des organes de reproduction. La meilleure densité de semis révélée par cette étude est largement supérieure à celle rapportée par Yang et al. (2014), qui a été de 3 plants $/ \mathrm{m}^{2}$, soit 30000 plants/ha ou la recommandation en vigueur en Inde qui était de 5,6 plants $/ \mathrm{m}^{2}$ ou 56000 plants/ha (Blaise \& Ravindran, 2003). Cependant, cette densité est inférieure aux recommandations en vigueur au Mali (8,3 à 25 plants $/ \mathrm{m}^{2}$ ou 83000 à 250000 plants/ha ; Barrabé et al. (2007), en Chine (22,5 plants/m² ou 225000 plants/ha; Han et al. (2009) ou aux Etats Unis (10 plants $/ \mathrm{m}^{2}$, équivalent à 100000 plants/ha ; Kawakami et al., 2012). De plus, les faibles densités favorisant la croissance végétative au détriment des organes de reproduction, ce qui peut entraîner du coup une baisse de rendement (Batamoussi et al., 2015).Par ailleurs, la présente étude n'a révélé aucune interaction significative entre la période et la densité de semis sur le rendement en coton graine suggérant que la réponse du cotonnier à la densité de semis n'a pas varié suivant les périodes de semis testées. Cependant, Awan et al. (2011) ont rapporté une interaction significative entre la période et la densité de semis pour le rendement en coton graine et ont recommandé un semis précoce avec un écartement de $20 \mathrm{~cm}$ entre plants au Pakistan.

Effet de la période et la densité de semis sur la morphologie du cotonnier: La période de semis a significativement affecté la hauteur des plants de cotonnier aux différentes dates d'observation sur les deux sites. Dans la zone centre-nord, les semis tardifs ont réduit le développement végétatif des cotonniers par rapport aux semis précoces et à bonne date (Tableau 5). Ces résultats sont en accord avec ceux rapportés par Sêkloka et al. (2015) qui ont montré que la période de semis a significativement affecté la morphologie des plants de cotonnier dans les conditions hydriques limitantes et les plants plus courts ont été observés sur les parcelles ayant accueillis les semis tardifs. Les mêmes observations ont été faites par Awan et al. (2011)

\section{CONCLUSION}

L'objectif de l'étude était d'identifier les meilleures périodes et densités de semis dans les différentes zones agro-écologiques cotonnières du Bénin dans un contexte de changement climatique. Au terme des deux années d'expérimentation, il a été révélé que le semis du cotonnier dans la zone nord peut s'étendre sur quatre qui ont rapporté que la hauteur de la plante a été affectée de manière significative par les différentes périodes de semis. Cependant, la densité de semis, n'a pas affecté la hauteur des plants sur les différents sites ayant abrité les essais. Cette observation est contraire aux résultats de Awan et al. (2011) qui ont rapporté de différence significative entre différentes densités de semis pour la hauteur des plants de cotonnier. II en est de même pour Sêkloka et al. (2015) qui ont rapporté la réduction de la taille des cotonniers semés à une forte densité.

Effet de la période et la densité de semis sur le shedding des organes de reproduction : Les résultats ont montré que le shedding des organes de reproduction (boutons floraux et capsules) sains a été plus important que celui des organes parasités à Aganradébou qu'à Okpara suggérant ainsi que la chute des organes de reproduction a été plus physiologique que parasitaire. La période de semis a également affecté la chute des organes de reproduction. Ces résultats peuvent être expliqués par les conditions environnementales qui ont prévalu au cours des différents essais. Un déficit hydrique pendant la période de fructification et une période prolongée d'humidité favorise le phénomène de shedding (Guinn, 1982; Vaughan \& Bate, 1977). Konan et al. (2015) ont également attribué la chute précoce des boutons floraux à un déficit hydrique pendant la période florifère et fructifère du cotonnier en Côte d'Ivoire. De plus, le cotonnier étant une plante qui préfère la lumière, une période prolongée de temps nuageux entraîne une augmentation temporaire de la chute des capsules (Guinn, 1982). Le shedding des organes de reproduction a été plus important sous la forte densité de semis. En effet, à la période active de développement végétatif, les fortes densités de semis ne favorisent pas la pénétration des rayons solaires dans le feuillage pour favoriser la synthèse des assimilas grâce à la photosynthèse pour la nutrition de ces organes de reproduction. Ces derniers chutent afin de favoriser le développement et le maintien d'autres organes. Konan et al. (2015) ont attribué la pourriture des capsules à la base des plants de cotonnier à un défaut de pénétration des insecticides et de la lumière à l'intérieur de la végétation lorsque la hauteur des plants dépasse $1,5 \mathrm{~m}$.

décades (20 mai au 30 juin) contrairement au centrenord où la période optimale de semis s'étend seulement sur les deux dernières décades de juin (10-30 juin). Ces résultats ont montré qu'on peut recommander pour la zone nord, en plus des trois décades de semis déjà en vulgarisation (20 mai au 20 juin), la dernière décade de 
juin. Par contre dans le centre-nord, il n'est pas utile de faire des recommandations de semis du coton durant le mois de juillet. Ces recommandations pourront évoluer lorsque la recherche cotonnière mettra au point des variétés précoces pour mieux gérer les perturbations climatiques. Par ailleurs, la nouvelle densité de semis identifiée peut être une option pour les producteurs qui

\section{RÉFÉRENCES BIBLIOGRAPHIQUES}

AIC. Association Interprofessionnelle du Coton du Bénin, 2017. Rapport Annuel Direction des opérations AIC campagne 2016-2017 [Rapport d'activité]. Cotonou, République du Bénin: Association Interprofessionnelle du Coton du Bénin.

AIC. Association Interprofessionnelle du Coton du Bénin, 2018. Rapport Annuel Direction des opérations AIC campagne 2017-2018 [Rapport d'activité]. Cotonou, République du Bénin: Association Interprofessionnelle du Coton du Bénin

AIC. Association Interprofessionnelle du Coton du Bénin, 2020. Rapport Annuel Direction des opérations AIC campagne 2019-2020 [Rapport d'activité]. Cotonou, République du Bénin: Association Interprofessionnelle du Coton du Bénin.

Akhtar M.E., Sardar A., Ashraf M., Akhtar M. et Khan M.Z., 2003. Effect of potash application on seed cotton yield and yield components of selected cotton varieties-I. Asian Journal of Plant Science 2(8): 602-604. Consulté à l'adresse http://docsdrive.com/pdfs/ansinet/ajps/2003/60 2-604.pdf

Ali M.A., Bashir S. et Ali L., 2004. Cotton yield as affected by different dates. International Journal of Agriculture and Biology, 6(4): 644-646.

Amonmidé I., Dagbenonbakin G., Agbangba C.E. et Akponikpe P., 2019. Contribution à l'évaluation du niveau de fertilité des sols dans les systèmes de culture à base du coton au Bénin. International Journal of Biological and Chemical Sciences, 13(3): 1846-1860. https://dx.doi.org/10.4314/ijbcs.v13i3.52

Arshad M., Wajid A., Maqsood M., Hussain K., Aslam M. et Ibrahim M., 2007. Response of growth, yield and quality of different cotton cultivars to sowing dates. Pakistan Journal of Agricultural Sciences, 44(2): 208-212.

Awan H., Awan I., Mansoor M., Khan E.A. et Khan M.A., 2011. Effect of sowing time and plant spacing pourront faire des opérations de semis et de fertilisation mécanisées, compte tenu de la distance inter-poquets qui est réduite par rapport aux deux densités en vulgarisation. Mais dans un système mécanisé, cette densité (62500 plants/ha: $0,80 \mathrm{~m} \times 0,20 \mathrm{~m})$ serait vivement conseillée.

on fiber quality and seed cotton yield. Sarhad Journal of Agriculture, 27(3), 411-413.

Banque Mondiale, 2016. Notes de politiques pour la nouvelle administration béninoise. Banque Mondiale (Ed), $145 \mathrm{p}$.

Barrabé A., Rapidel B., Sissoko F., Traoré B. et Wery J., 2007. Elaboration and test of a decision rule for the application of mepiquat chloride on cotton in Mali. European Journal of Agronomy, 27(2-4), 197-204. https://doi.org/10.1016/j.eja.2007.04.001

Batamoussi H.M., Moumouni I., et Mere, S.B.J.T.O., 2015. Contribution à l'amélioration des pratiques paysannes de production durable de coton (Gossypium hirsutum) au Bénin : Cas de la commune de Banikoara. International Journal of Biological and Chemical Sciences, 9(5): 2401-2413.

Bilal M.F., Saleem M.F., Wahid M.A., Saeed A., et Anjum S.A., 2015. Varietal comparison of Bt and nonBt cotton (Gossypium hirsutum L.) under different sowing dates and nitrogen rates. Soil \& Environment, 34(1). 34-43

Blaise D., et Ravindran C.D., 2003. Influence of tillage and residue management on growth and yield of cotton grown on a vertisol over 5 years in a semi-arid region of India. Soil and Tillage Research, $\quad$ 70(2): 163-173. https://doi.org/10.1016/S0167-1987(02)001538

Bozbek T., Sezener V., et Unay, A., 2006. The effect of sowing date and plant density on cotton yield. Journal of Agronomy, 5(1): 122-125. DOI: 10.3923/ja.2006.122.125

Ciampitti I.A. et Vyn T.J., 2011. A comprehensive study of plant density consequences on nitrogen uptake dynamics of maize plants from vegetative to reproductive stages. Field Crops Research, $\quad$ 121(1), 2-18. https://doi.org/10.1016/j.fcr.2010.10.009

De Bruin J.L. et Pedersen P., 2008. Soybean seed yield response to planting date and seeding rate in 
the Upper Midwest. Agronomy Journal, 100(3), 696-703. DOI: 10.2134/agronj2007.0115

Djihinto C.A., Affokpon A., Danon E. et Aboua C., 2016. Réduction de doses de cyperméthrinetriazophos et lutte raisonnée en culture cotonnière au Bénin. Journal of Applied Biosciences, $\quad 98(0)$, 9261-9269. https://doi.org/10.4314/jab.v98i1.3

Dong H., Li W., Eneji A.E. et Zhang D., 2012. Nitrogen rate and plant density effects on yield and lateseason leaf senescence of cotton raised on a saline field. Field Crops Research, 126: 137-144. https://doi.org/10.1016/ j.fcr. 2011.10 .005

FAOSTAT (2020). FAOSTAT database. Food and Agriculture Organization of the United Nations, Rome, Italy, 1. http://www.fao.org/faostat/fr/\#data/QC, Consulté le 14 Août 2020.

Guinn G., 1982. Causes of square and boll shedding in cotton. US Dept. of Agriculture, Agricultural Research Service.

Han H.Y., Deng F.J., Li B.C., Yang B.Y., Yang, L. Y., Lin, H., et Wang X.T., 2009. Effect of plant density on cotton yield and quality in Xinjiang. Jiangsu Journal of Agricultural Science, $4: 98-100$.

Hougni A., Imorou L., Dagoudo A. et Zoumarou-Wallis N., 2016. Caractérisation agro-morphologique de variétés de cotonnier (Gossypium hirsutum L.) pour une régionalisation économique pour la production du coton au Benin. European Scientific Journal, 36(12), 210-227. http://dx.doi.org/10.19044/esj.2016.v12n36p21 0

Igue A.M., Saidou A., Adjanohoun A., Ezui G., Attiogbe P., Kpagbin, G., ... Balogoun, I., 2013. Evaluation de la fertilité des sols au sud et centre du Bénin. Bulletin de Recherches Agronomiques du Bénin, Spécial numéro, Fertilisation du maïs, 12-23.

INSAE, Institut National de la Statistique et de l'Analyse Economique, 2019. Les statistiques agricoles. Consulté 26 septembre 2019, à l'adresse INSAE, Institut National de la Statistique et de l'Analyse Economique website: https://www.insae-

bj.org/statistiques/statistiques-economiques

Jagannathan N.T. et Venkitaswamy R., 1996. Effect of plant density and nutrient levels on new cotton varieties. Madras Agricultural Journal, 83 : 159160.
Kakar N.U., Oad, F.C., Tunio S., Chachar Q.U. et Kandhro, M.M., 2012. Response of sowing time to various cotton genotypes. Sarhad J. Agric, 28(3): 379-385.

Kawakami E.M., Oosterhuis D.M., Snider J.L., et Mozaffari M., 2012. Physiological and yield responses of field-grown cotton to application of urea with the urease inhibitor NBPT and the nitrification inhibitor DCD. European Journal of Agronomy, $\quad 43$ : 147-154. https://doi.org/10.1016/j.eja.2012.06.005

Khan K., Mahmood Z., Soomro A.R. et Illahi N., 2005. Seed cotton yield as influenced by different plant spacings under DI Khan environment. The Indus Cottons, 2.

Konan J.N., Fofana I.J., Silue S., Diarrassouba N., N'guessan E. et Sangare A., 2015. Caractérisation agromorphologique précoce de quarante-huit lignées de cotonnier (Gossipium hirsutm L.) en Côte d'Ivoire. Afrique Science, 11(5): 422-432.

Muhammad D.M., Anwar M., Zaki M. S., et Afzal M.N., 2002. Effect of plant population and nitrogen variables on cotton crop. The Pak. Cottons, 47(1 \& 2) : 37-41.

PAM. Programme Alimentaire Mondial des Nations Unies, 2014. Analyse Globale de la Vulnérabilité et de la Sécurité Alimentaire (AGVSA), République du Bénin. Via C.G. Viola 68, Parco de Medici, 00148, Rome, Italie : Programme Alimentaire Mondial des Nations Unies, 146p.

PNUD, Programme des Nations Unies pour le Développement, 2011. Stratégie de croissance pour la réduction de la pauvreté (SCRP 20112015).

Sêkloka E., Lançon J., Batamoussi M. et Thomas G., 2015. La réduction de la croissance végétative à forte densité de semis comme stratégie d'adaptation variétale aux semis tardifs en culture cotonnière pluviale au Bénin. Tropicultura, 33(4): 299-308.

Shah S.M., Khan M.S.S., Sajjad T. et Malik W., 2005. Effect of various plant populations on seed cotton yield and other yield components. The Indus Cottons, 2.

Soomro A.R., Channa M.H., Channa A.A., Kalwar G.H., Dayo G.N. et Memon A.H., 2000. The effect of different sowing dates on the yield of newly developed strain under climatic conditions of 
Ghotki, Sindh. Pakistan Journal of Biological Sciences, 3(11): 1901-1903.

Vaughan A.K.F. et Bate G.C., 1977. Changes in the levels of ethylene, abscisic acid like substances and total nonstructural carbohydrate in young cotton bolls in relation to abscission induced by a dark period. Rhod Journal of Agricultural Research 15: 51-63.

Yang G., Luo X., Nie Y. et Zhang X., 2014. Effects of Plant Density on Yield and Canopy Micro Environment in Hybrid Cotton. Journal of Integrative Agriculture, 13(10): 2154-2163. https://doi.org/10.1016/S2095-3119(13)607273 